\title{
Constraining active-sterile neutrino transition magnetic moments at DUNE near and far detectors
}

\author{
Thomas Schwetz, ${ }^{a}$ Albert Zhou ${ }^{a}$ and Jing-Yu Zhu ${ }^{a, b}$ \\ ${ }^{a}$ Institut für Astroteilchen Physik, Karlsruher Institut für Technologie (KIT), \\ Hermann-von-Helmholtz-Platz 1, 76344 Eggenstein-Leopoldshafen, Germany \\ ${ }^{b}$ School of Physics and Astronomy and Tsung-Dao Lee Institute, Shanghai Jiao Tong University, \\ Shanghai 200240, China \\ E-mail: schwetz@kit.edu, albert.zhou@kit.edu, jing-yu.zhu@kit.edu
}

ABSTRACT: We consider the sensitivity of the DUNE experiment to a heavy neutral lepton, $\mathrm{HNL}$ (also known as sterile neutrino) in the mass range from a few $\mathrm{MeV}$ to a few $\mathrm{GeV}$, interacting with the Standard Model via a transition magnetic moment to the active neutrinos, the so-called dipole portal. The HNL is produced via the up-scattering of active neutrinos, and the subsequent decay inside the detector provides a single-photon signal. We show that the tau-neutrino dipole portal can be efficiently probed at the DUNE far detector, using the tau-neutrino flux generated by neutrino oscillations, while the near detector provides better sensitivity to the electron- and muon-neutrino dipole portal. DUNE will be able to explore large regions of currently unconstrained parameter space and has comparable sensitivity to other planned dedicated experiments, such as SHiP. We also comment briefly on the sensitivity to pure HNL mixing with the tau neutrino at the DUNE far detector.

Keywords: Beyond Standard Model, Neutrino Physics

ArXiv EPrint: 2105.09699 


\section{Contents}

1 Introduction 1

2 Dipole decay signal at DUNE $\quad 3$

2.1 Inside-event rate 4

2.2 Outside-event rate 5

$\begin{array}{lll}2.3 & \text { Example spectra } & 6\end{array}$

3 Results $\quad 6$

3.1 DUNE-FD sensitivity to $d_{\tau} \quad 6$

3.2 Near-detector sensitivity to the $\nu_{e}$ and $\nu_{\mu}$ dipole portal $\quad 9$

$\begin{array}{lll}3.3 \text { Global picture } & 10\end{array}$

4 DUNE-FD sensitivity to active-sterile mixing via $U_{\tau 4} \quad 14$

$\begin{array}{llr}5 & \text { Conclusion } & 16\end{array}$

$\begin{array}{ll}\text { A Cross sections } & 17\end{array}$

$\begin{array}{ll}\text { B Inside-event rate integral } & 19\end{array}$

$\begin{array}{ll}\text { C Outside-event rate integral } & 21\end{array}$

$\begin{array}{ll}\text { D Estimate of the near-detector signal } & 23\end{array}$

\section{Introduction}

Neutrino mass requires an extension of the Standard Model (SM). Many known mechanisms to give mass to neutrinos involve fermionic SM-gauge-group singlets, so-called sterile neutrinos, also referred to as heavy neutral leptons (HNL). However, in general there are no specific indications about the mass scale of such sterile neutrinos. Moreover, it is possible that they act as a portal to generic new physics. In this paper we will assume that a $\mathrm{HNL}$, denoted by $\nu_{4}$, exists in the mass range from a few $\mathrm{MeV}$ to a few $\mathrm{GeV}$, and that it interacts with the SM via a transition magnetic moment - the so-called "dipole portal" - described by the following term in the Lagrangian:

$$
\mathcal{L}=d_{\alpha} \bar{\nu}_{\alpha L} \sigma^{\mu \nu} \nu_{4} F_{\mu \nu}+\text { h.c. }
$$

Here, $\nu_{\alpha}$ is a left-handed SM neutrino field of flavour $\alpha=e, \mu, \tau, F_{\mu \nu}$ is the photon field strength tensor, and $\sigma^{\mu \nu}=\frac{i}{2}\left(\gamma^{\mu} \gamma^{\nu}-\gamma^{\nu} \gamma^{\mu}\right)$ is the antisymmetric combination of Dirac gamma matrices. 
Eq. (1.1) corresponds to an effective Lagrangian, valid up to a cut-off energy scale $\Lambda$, where the transition magnetic moment $d_{\alpha}$ is expected to be of order $1 / \Lambda$. Note that eq. (1.1) is not $\mathrm{SU}(2)_{L}$ gauge invariant and therefore $1 / \Lambda \sim v / \Lambda^{\prime 2}$, with $v$ denoting the Higgs vacuum expectation value. A discussion on some model-building aspects can be found in [1], see also [2]. In this work we will remain agnostic about the UV origin of this operator and study its phenomenological implications at energies small compared to the electro-weak scale $v$.

The heavy-neutrino dipole portal has been investigated by a number of authors. Comprehensive reviews of various laboratory, astrophysical, and cosmological bounds on $d_{\alpha}$ can be found in refs. [1,3]. Other studies include considerations of solar neutrinos [4-6], atmospheric neutrinos in IceCube [7], or short-baseline experiments [8-13]. The HNL dipole portal can be explored also at dedicated experiment for long-lived particle searches such as SHiP [14], FASER [15, 16], or MATHUSLA [17, 18].

Typically it is difficult to test a transition moment between the tau neutrino and a HNL, $\alpha=\tau$ in eq. (1.1), since it is hard to produce an intense $\nu_{\tau}$ flux. In this work we will exploit neutrino oscillations to overcome this problem: we consider $\nu_{\mu} \rightarrow \nu_{\tau}$ oscillations at the DUNE long-baseline experiment [19], governed by a transition amplitude of order one. These $\nu_{\tau}$ may up-scatter on nuclei, nucleons or electrons to a HNL via the dipole interaction eq. (1.1). The heavy neutrino can travel over macroscopic distances and decay back into a light neutrino and a photon inside the detector. Below we will calculate the sensitivity of the DUNE far detector to $d_{\tau}$ using these processes. Due to the sizeable primary $\nu_{\mu}$ and $\nu_{e}$ fluxes, the HNL transition moments $d_{\mu}$ and $d_{e}$ are more efficiently probed at the near detector. We will also provide estimates of the DUNE near detector sensitivities from the

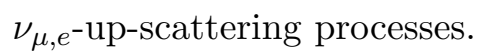

The outline of our paper is as follows. In section 2 we discuss the general features of the signal considered in this paper and provide an outline of the relevant event-rate calculations. Section 3 contains our main results: in section 3.1 we show the sensitivity of the DUNE far detector to the tau-neutrino dipole portal, whilst section 3.2 contains the near-detector sensitivities to the electron- and muon-neutrino dipole portal. In section 3.3 we set the DUNE sensitivities in the context of various laboratory and astrophysical constraints from the literature, showing that DUNE will cover large currently unconstrained regions in parameter space, and is competitive with prospective sensitivities from the SHiP experiment [14]. In most parts of this paper we will assume that HNL mass mixing with active flavours is negligible and the dipole interaction dominates. However in section 4 we briefly comment on the sensitivity of the DUNE far detector to HNL mixing with $\nu_{\tau}$ : we find that the far detector sensitivity is somewhat weaker than the sensitivity of the near detector from the prompt $\nu_{\tau}$ flux. We conclude in section 5. In appendix A we summarize the cross section formulae relevant for the HNL up-scattering mediated by the dipole portal. Appendices $\mathrm{B}$ and $\mathrm{C}$ provide technical details on the event-rate calculations. In appendix D we estimate the event rate at the near detector from the tau-neutrino dipole portal, indicating that the signal at the far detector dominates.

Throughout this article, the HNL decay width we use applies in the case of a Dirac sterile neutrino. A Majorana neutrino will have twice as large a decay width [20]. A treatment of their interesting differences can be found in [20-22]. 

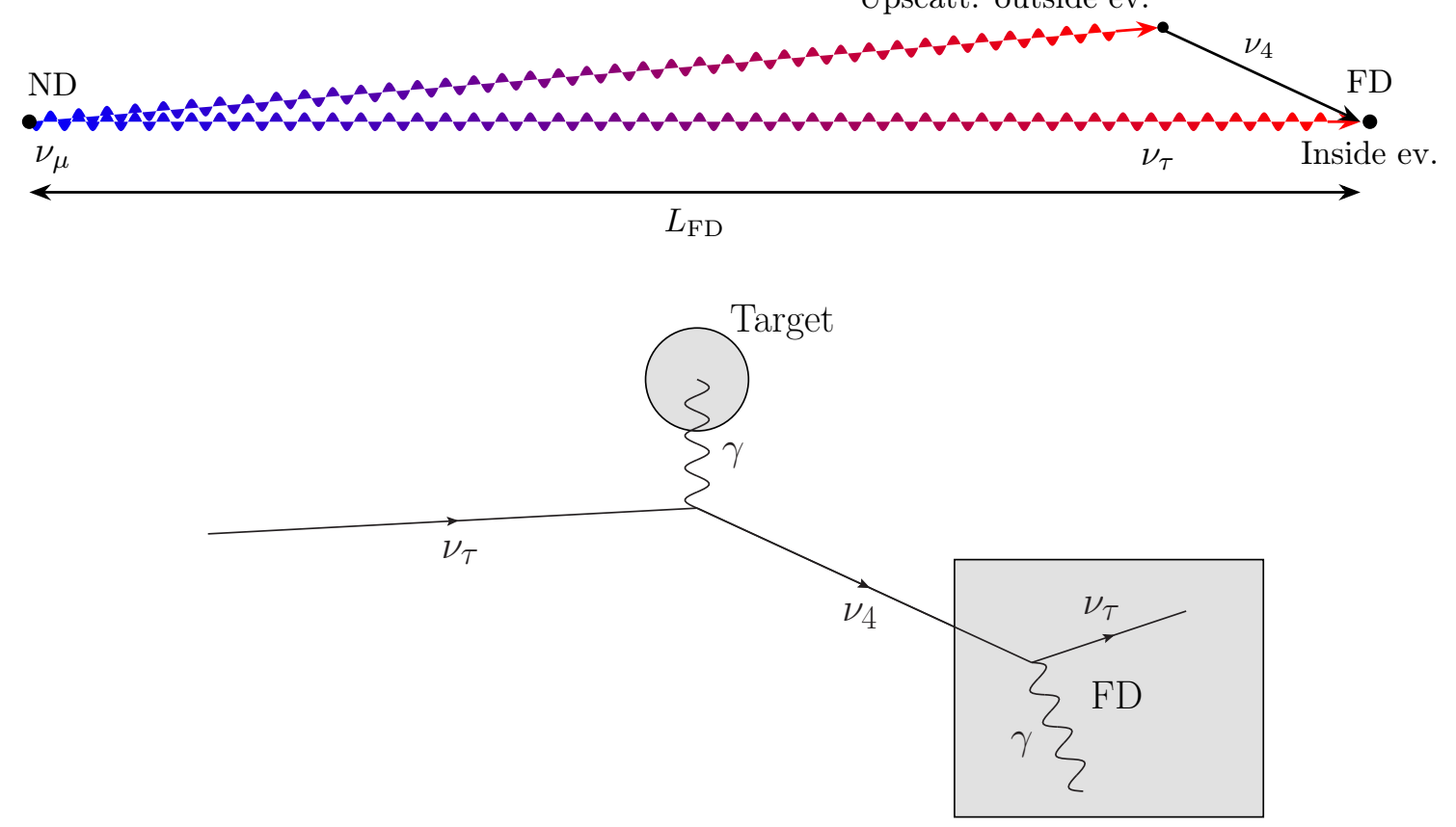

Figure 1. Cartoons of the up-scattering production and decay signal for $d_{\tau}$ in the far detector.

\section{Dipole decay signal at DUNE}

The DUNE experiment produces a flux of mostly muon neutrinos with a subleading component of electron neutrinos. These fluxes can be used to directly search for $d_{e}$ and $d_{\mu}$ at the DUNE near detector (ND). However, to constrain a tau transition moment $d_{\tau}$, we use the muon neutrinos, which oscillate into tau neutrinos during their propagation through the Earth. ${ }^{1}$ Tau neutrinos can up-scatter off target particles in the Earth (protons, electrons or coherently on nuclei) and can be converted via the dipole transition moment into a sterile neutrino. If the up-scattering occurs outside the detector in the Earth's crust or upper mantle, and if the sterile-neutrino mass is low enough, the sterile neutrino will be long lived and can travel through the Earth and decay inside the DUNE far detector (FD). We call these outside events. If the sterile neutrino has a large enough mass and/or dipole moment, both up-scattering and decay can occur inside the detector. We call these inside events. The sequence of events is illustrated in figure 1 .

We consider coherent scattering off nuclei in the Earth and incoherent scattering off protons, neutrons and electrons. Note that, since neutrons can only interact magnetically, as they have no electric charge, their cross section is suppressed by a factor $\eta \equiv Q^{2} /\left(4 M_{T}^{2}\right)$. Our expressions come from [1, appendix A] and [3, eqs. (4,5)], and are summarised for completeness in appendix A. For neutral-current (NC) scattering, the cross-section for electrons can be neglected, since the total cross section scales linearly with the mass of the

\footnotetext{
${ }^{1}$ The primary flux of tau neutrinos in the beam has been estimated recently in [22-24]. We have estimated that the sensitivity of the $d_{\tau}$-induced event rate in the ND is significantly smaller than the one in the FD discussed here, see section 3.2 and appendix D for further discussions.
} 
target particle, see also [25, figure 8]. However, the total dipole cross section goes logarithmically with the target-particle mass, so electrons can give a relevant contribution (see below). For the coherent cross section, we consider Argon nuclei for the events inside the detector, and for the outside events the nuclei: oxygen, silicon, aluminium, iron, calcium, sodium, magnesium, potassium, titanium; whose abundances we take from [26].

Therefore, depending on the different event classes, we expect the following signatures in the detector:

- Outside events: as the scattering happens outside the detector, the signature is a single-photon event.

- Inside events, coherent: the coherent scattering on the nucleus leaves the nucleus intact and provides a nuclear recoil of low energy, which is difficult to observe in the detector (see however $[27,28]$ ). Thus, the signal is again a single-photon event.

- Inside events, incoherent: the incoherent scattering on nucleons leads to a signature similar to NC neutrino events, whereas the scattering on electrons results in a single electron. Hence, the signature will be either a NC-like or single-electron type event in coincidence with the displaced single-photon event from the heavy-neutrino decay.

While the first two event classes provide a clean single-photon signal, the last event category offers a richer signature which can be explored by dedicated analyses for improved background discrimination. In this work we will consider all event types on equal footing and assume for all of them the single-photon reconstruction efficiency given in [19] (see appendix B for further details). Liquid-argon detectors can discriminate electrons and photons very well [29-31]; so the relevant backgrounds for the dipole signal are the singlephoton process $\mathrm{NC} 1 \gamma$ and highly asymmetric $\mathrm{NC} \pi^{0}$-decays, where the two photons from the pion decay cannot be distinguished. Single-photon production from neutral-current scattering $(\mathrm{NC} 1 \gamma)$ is expected to have a total cross section $\sim 10^{-41} \mathrm{~cm}^{2} /$ nucleon $[32-34]$. We estimate that this process would induce a background of 0.1 events/year (see [35] for a $\mathrm{NC} 1 \gamma$ analysis in $\mathrm{T} 2 \mathrm{~K}$ ). Therefore, photons from $\mathrm{NC} \pi^{0}$ are expected to be the dominant background, see $[30,31]$ for studies in liquid argon detectors. A detailed detector simulation including analysis cuts is beyond the scope of this work and we concentrate on predicting the expected signal rate.

Below we outline the calculation of the event rate. We focus first on the $d_{\tau}$-induced signal in the FD and comment on the relevant modification for the $d_{\mu, e}$ signal in the ND in section 3.2. We use the indices $\nu, 4, T$, and $\gamma$ to denote the initial light neutrino, the heavy sterile neutrino, the scattering target, and the final photon, respectively. The target $T$ can be an electron, proton, neutron, or a nucleus (for coherent interactions).

\subsection{Inside-event rate}

Let a sterile neutrino be produced at the location $\vec{x}_{p}$ and decay at $\vec{x}_{d}$, both inside the detector volume $V_{D}$; let $\ell_{d}$ be the maximum decay length, when $\vec{x}_{d}$ is at the edge of the detector. The decay direction (which defines the solid angle $\left.\Omega_{s}\right)$ is $\left(\vec{x}_{d}-\vec{x}_{p}\right) / \ell_{d}$. We wish 
to calculate the following integral for the differential event rate from a given target type $T$ :

$$
\frac{\mathrm{d} N_{T}}{\mathrm{~d} E_{\nu}}=N_{\text {mod }} \int_{V_{D}} \mathrm{~d}^{3} \vec{x}_{p} \frac{L_{\mathrm{ND}}^{2}}{\left|\vec{x}_{p}\right|^{2}} \frac{\mathrm{d}^{2} \Phi}{\mathrm{d} \Omega \mathrm{d} E_{\nu}} P_{\text {osc }}\left(\frac{\left|\vec{x}_{p}\right|}{E_{\nu}}\right) \rho_{N}\left(\vec{x}_{p}\right) \int \mathrm{d} \Omega_{s} \frac{\mathrm{d} \sigma_{T}}{\mathrm{~d} \Omega_{s}} P_{\operatorname{dec}}\left(\ell_{d}\right) \varepsilon\left(p_{4}\right) .
$$

From left to right, the terms on the r.h.s. are the number of detector modules $N_{\text {mod }}=4$, an integral of the up-scattering location, the geometric suppression, the differential $\nu_{\mu}$ flux $\Phi$ at the near-detector location $L_{\mathrm{ND}}$, the $\nu_{\mu} \rightarrow \nu_{\tau}$ oscillation probability $P_{\mathrm{osc}}$, the nucleon density $\rho_{N}$, the integral over the scattering solid angle, the cross section per solid angle per nucleon, the probability $P_{\mathrm{dec}}$ that the heavy neutrino decays inside the detector, and the photon reconstruction efficiency $\varepsilon$ evaluated as a function of the heavy-neutrino momentum. The energy of the light neutrino is $E_{\nu}$, whereas $E_{4}$ and $p_{4}$ denote the energy and momentum of the heavy neutrino. Details of all these terms may be found in appendices A and B.

We make a series of approximations, which are also detailed in appendix B. Briefly, as the baseline is much larger than the detector dimensions we set $\left|\vec{x}_{p}\right|=L_{\mathrm{FD}}$ and assume the neutrino beam is collimated (i.e. on-axis), so there is no angular dependence of the flux. We also take the detector nucleon density $\rho_{N}$ to be constant (whose value is determined by the detector density).

By assuming the detector is cylindrical and using cylindrical coordinates $\left(\rho, \theta_{p}, z\right)$, the polar-angle dependence, $\theta_{p}$, of the integrand drops out. By assuming the decay length $\ell_{d}$ can be approximated by its value at the centre $\ell_{d}(\rho=0) \equiv \ell_{d}^{0}$, the $\rho$ - and $\varphi_{s}$-integrals can be done analytically. This approximation becomes exact in the limit of large decay width (as all decays happen instantaneously so $\ell_{d}$ becomes irrelevant), but when the decay-length is comparable to or larger than the detector size there should be a penalty term $\Pi\left(\ell_{d}^{0}\right)$ due to the geometry; this is precalculated (see appendix B). With this simplifying assumption, the $\rho-\theta_{p}$ integral $\iint_{A} \rho \mathrm{d} \rho \mathrm{d} \theta_{p}$ may simply be replaced with the detector cross-section area $A_{\text {det }}$. The final expression, summed over the scattering targets $T$, is then

$$
\begin{aligned}
\frac{\mathrm{d} N}{\mathrm{~d} E_{\nu}}= & \left.N_{\mathrm{mod}} \frac{L_{\mathrm{ND}}^{2}}{L_{\mathrm{FD}}^{2}} \rho_{N} A_{\mathrm{det}} \frac{\mathrm{d} \Phi}{\mathrm{d} \Omega \mathrm{d} E_{\nu}}\right|_{\theta_{b}=0} P_{\mathrm{osc}}\left(\frac{L_{\mathrm{FD}}}{E_{\nu}}\right) \times \\
& \sum_{M_{T}} \int_{0}^{L_{d}} \mathrm{~d} z \int_{-1}^{1} \mathrm{~d} \cos \theta_{s} \frac{\mathrm{d} \sigma_{T}}{\mathrm{~d} \cos \theta_{s}} \Pi\left(\ell_{d}^{0}\right) P_{\mathrm{dec}}\left(\ell_{d}^{0}\right) \varepsilon\left(p_{4}\right) .
\end{aligned}
$$

\subsection{Outside-event rate}

We start from eq. (2.1), which also applies to the outside events; however we will now integrate $\vec{x}_{p}$, the $\nu_{4}$ production point, over the region of the Earth exposed to the neutrino beam including the off-axis beam [36, 37]. In addition, the nucleon density is assumed to be constant below sea level and zero above sea level. Inside the Earth, we take $2.9 \mathrm{~g} / \mathrm{cm}^{3}$ as the mass density, which is roughly the surface density (although at $\theta_{b}^{\max } \approx 3.6^{\circ}$ the density can reach $3.375 \mathrm{~g} / \mathrm{cm}^{3}[38]$ ).

Note that the geometry of the Earth breaks the otherwise cylindrical symmetry around the beam axis. We denote the coordinates of the scattering point in spherical coordinates $\left(r_{p}, \theta_{b}, \varphi_{b}\right)$; then the boundary of the earth imposes the boundary conditions on the $\varphi_{b}$-integral: $\varphi_{b} \in\left[\Phi\left(r_{p}, \theta_{b}\right), 2 \pi-\Phi\left(r_{p}, \theta_{b}\right)\right]$ (see figure 10 in appendix C). Since the 
rest of the integrand has no $\varphi_{b}$-dependence, we replace the $\varphi_{b}$-integral with the factor $\varepsilon_{\varphi_{b}} \equiv 2\left[\pi-\Phi\left(r_{p}, \theta_{b}\right)\right]$. Finally, we assume the geometry of the detector can be neglected. Therefore, $\theta_{s}$ and $\varphi_{s}$ are uniquely determined by the production point $\vec{x}_{p}$. We replace the inner integral with an estimate of the solid angle $\Delta \Omega_{s}$ of the detector as viewed from $\vec{x}_{p}$. Note that the decay probability also changes to account for the distance required to travel to the detector. For expediency, when the phase of the oscillation probability is large, we replace $P_{\text {osc }}$ with $1 / 2$, assuming the wiggles average out; this was checked to only negligibly change the result compared to the full integral. The final expression is

$$
\begin{aligned}
\frac{\mathrm{d} N}{\mathrm{~d} E_{4}}= & N_{\bmod } \frac{\rho_{N}}{2 \pi} \int_{0}^{\theta_{b}^{\max }} \sin \theta_{b} \mathrm{~d} \theta_{b} \int_{r_{\min }}^{r_{\max }} L_{\mathrm{ND}}^{2} \mathrm{~d} r_{p} \times \\
& \sum_{M_{T}}\left[\frac{\mathrm{d}^{2} \Phi}{\mathrm{d} \Omega_{b} \mathrm{~d} E_{\nu}} \frac{\mathrm{d} E_{\nu}}{\mathrm{d} E_{4}} P_{\mathrm{osc}}\left(\frac{r_{p}}{E_{\nu}}\right) \varepsilon_{\varphi_{b}}\left(r_{p}, \theta_{b}\right) P_{\text {decay }}(\ell) \frac{\mathrm{d} \sigma}{\mathrm{d} \cos \theta_{s}} \Delta \Omega_{s} \varepsilon\left(p_{4}\right)\right]_{T} .
\end{aligned}
$$

The evaluation of $\varepsilon_{\varphi_{b}}$, as well as more details on the off-axis flux, geometric relations and approximations can be found in appendix $\mathrm{C}$; kinematic relations can be found in appendix A. We use the same reconstruction efficiency given in appendix B.

\subsection{Example spectra}

In figure 2 , we show example spectra for heavy-neutrino masses $M_{4}=10^{-3}, 10^{-2}, 10^{-1}, 1 \mathrm{GeV}$ for inside events and $M_{4}=10^{-3}, 10^{-2}, 5 \times 10^{-2} \mathrm{GeV}$ for outside events. For low energies (dashed), we replace the oscillation probability with $1 / 2$ to account for the averaging of fast oscillations. For the inside events this is only done for the plots shown and is not used for the numerical analysis, whereas for the outside events this significantly speeds up calculations and has a negligible impact on the final result.

We show inside events as a function of light-neutrino energy $E_{\nu}$, whereas the outside events as a function of the heavy-neutrino energy $E_{4}$. This is due to calculational convenience. ${ }^{2}$ The total number of events is then obtained by integrating over these spectra. We remark that for the lowest masses $M_{4} \sim 1 \mathrm{MeV}$, there is a sharp peak at low energies. This is due to the $1 / Q^{2}$ term in the cross section. The DUNE reconstruction efficiency for photons remains high at low photon momentum $\left(0.7\right.$ at $\left.p_{N}=0.1 \mathrm{GeV}[19]\right)$. For this reason, we do not cut off the spectrum at low energies and instead just fold in the reconstruction efficiency, see appendix B.

\section{Results}

\subsection{DUNE-FD sensitivity to $d_{\tau}$}

In figure 3 we show the six-events/year curve for inside (solid) and outside (dashed) events at the DUNE far detector for coherent scattering on nuclei (red), incoherent scattering on nucleons (blue) as well as electrons (purple) as a function of the heavy-neutrino mass $M_{4}$

\footnotetext{
${ }^{2}$ For inside events, the flux and oscillation probability (as functions of $E_{\nu}$ ) can be factored out of the inner integrals, whereas for the outside events, we use the more convenient expression of $E_{\nu}\left(E_{N}\right)$ see eqs. (2.2) and (2.3).
} 
Inside spectra with arbitrary normalisation

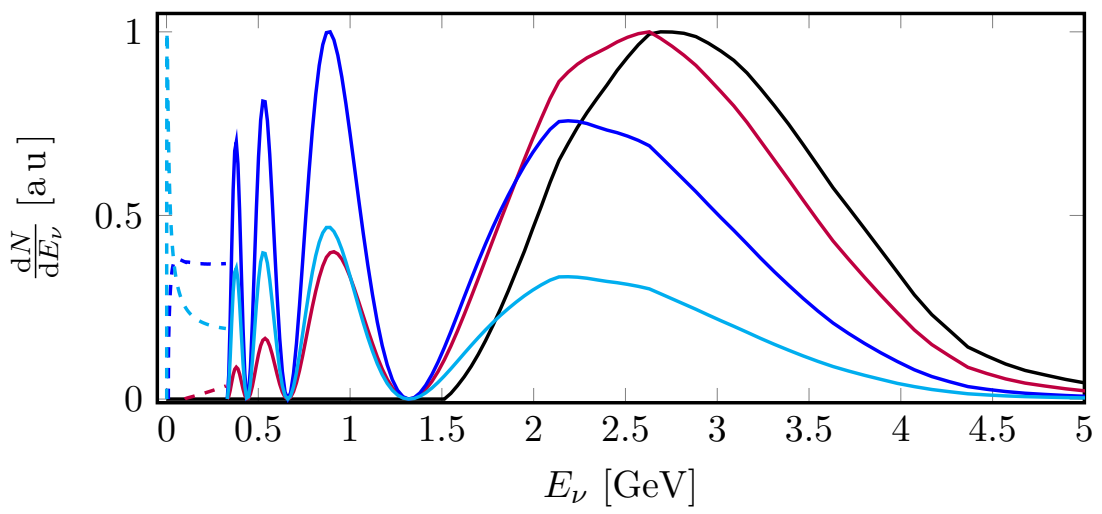

$$
\begin{aligned}
M_{4} & =1 \mathrm{GeV} \\
M_{4} & =10^{-1} \mathrm{GeV} \\
M_{4} & =10^{-2} \mathrm{GeV} \\
M_{4} & =10^{-3} \mathrm{GeV}
\end{aligned}
$$

Outside spectra with arbitrary normalisation

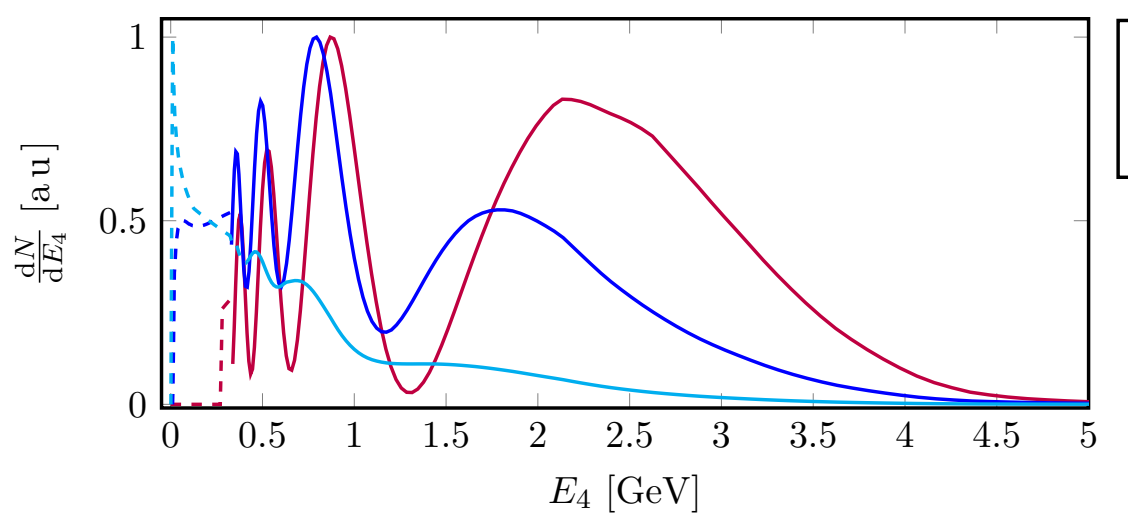

$\square M_{4}=5 \times 10^{-2} \mathrm{GeV}$
$M_{4}=10^{-2} \mathrm{GeV}$
$M_{4}=10^{-3} \mathrm{GeV}$

Figure 2. Spectra for inside and outside events at various masses, normalised so that the peak is 1. At low energies (dashed), we replace the oscillation probability with $1 / 2$ to account for the averaging of fast oscillations.

and transition magnetic moment $d_{\tau}$. Due to the lightness of the electron, up-scattering is kinematically allowed for low masses only, hence why the corresponding curve for inside events cuts off at $M_{4} \sim 0.077 \mathrm{GeV}$. The coherent scattering dominates until $M_{4} \sim 0.7 \mathrm{GeV}$, after which incoherent scattering off nucleons dominates. However above $1 \mathrm{GeV}$ the available beam energy rapidly kills the number of events.

For the outside events, the upper parts of the curves are indicative only. As $d_{\tau}$ increases, the decay length $1 / \Gamma$ decreases, so that more events occur close to the detector. At some point the events become too clustered around the detector, such that either the integrator cannot resolve the sharp peak and/or our assumptions break down (we begin to resolve the detector geometry). The cyan curve in the figure indicates when the decay length is $20 \mathrm{~m}$ at $E_{4}=1 \mathrm{GeV}$; for $d_{\tau}$ roughly above this line a full treatment of the detector geometry would be needed. Since inside events will dominate in this region anyway, we safely ignore this point.

DUNE will have sub-centimetre spatial resolution [39]. The grey curve in figure 3 corresponds to the case where the decay length for a HNL with energy $1 \mathrm{GeV}$ is $1 \mathrm{~cm}$. 
Inside (solid) and outside (dashed) 6 events/year at DUNE FD

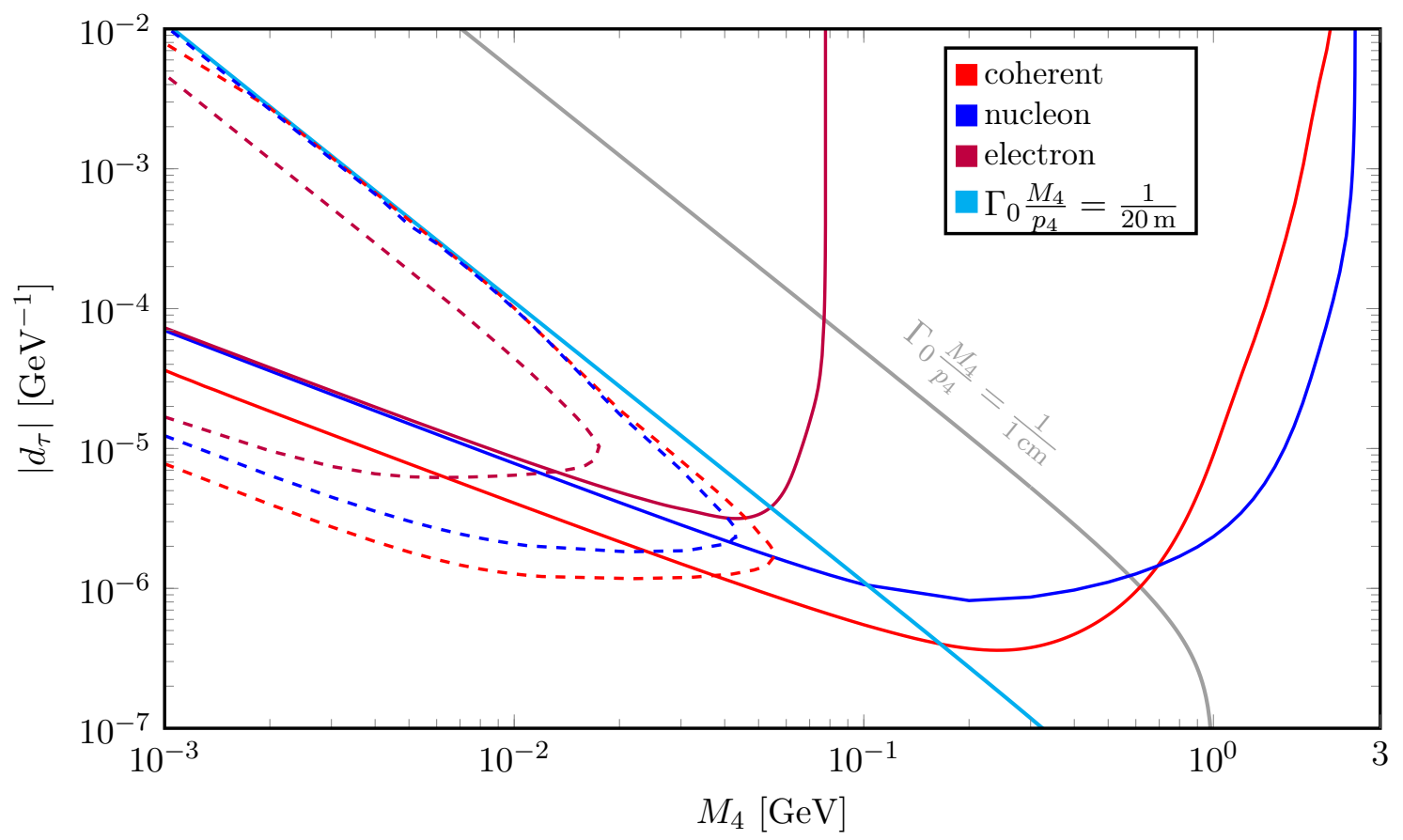

Figure 3. The 6-events/year curve for inside (solid) and outside (dashed) events at the DUNE FD for coherent scattering on nuclei (red), incoherent scattering on nucleons (blue) as well as electrons (purple). Near the upper curves of the outside events, up-scattering occurs close to the detector and our approximations break down, as indicated by the cyan line, at which the decay length is $20 \mathrm{~m}$ for $E_{4}=1 \mathrm{GeV}$. This effect is negligible as inside-events will dominate. We also show in grey when the decay-length is of the order of DUNE's spatial resolution [39].

Hence, in the region roughly below the grey curve it will be possible to resolve the displaced vertices of the hadronic signal and the single-photon decay for inside events. Note that the cyan and grey curves are only indicative, since they are for fixed HNL energy of $E_{4}=1 \mathrm{GeV}$ and do not take into account the energy spectrum.

Finally, we sum up the contributions from all event classes and show in figure 4 the curves corresponding to 2, 6, and 20 signal events per year. We compare these curves to the $95 \%$ confidence-level sensitivities from the SHiP experiment. SHiP [14] is a proposed experiment at the Super Proton Synchrotron (SPS) at CERN. Its sensitivity to the tauneutrino's dipole transition moment was evaluated in [1]. These authors consider two type of detectors, an emulsion cloud chamber (ECC) close to the beam target, as well as the "main" detector and calculate the $95 \%$ C.L. sensitivity after 5 years of running $\left(2 \times 10^{20}\right.$ POT [40]), assuming 100 background events in both detectors. For comparison, the curves shown for the DUNE FD correspond to 10, 30 and 100 events in 5 years; using signal $/ \sqrt{\text { background }} \approx 2$ this would give a 95\% C.L. sensitivity for 25,225 and 2500 background events, respectively.

In figure 4 we also compare our DUNE sensitivity with an exclusion estimate for the IceCube detector due to so-called double-bang events, induced by heavy neutrinos produced 
DUNE FD vs. SHiP \& IceCube

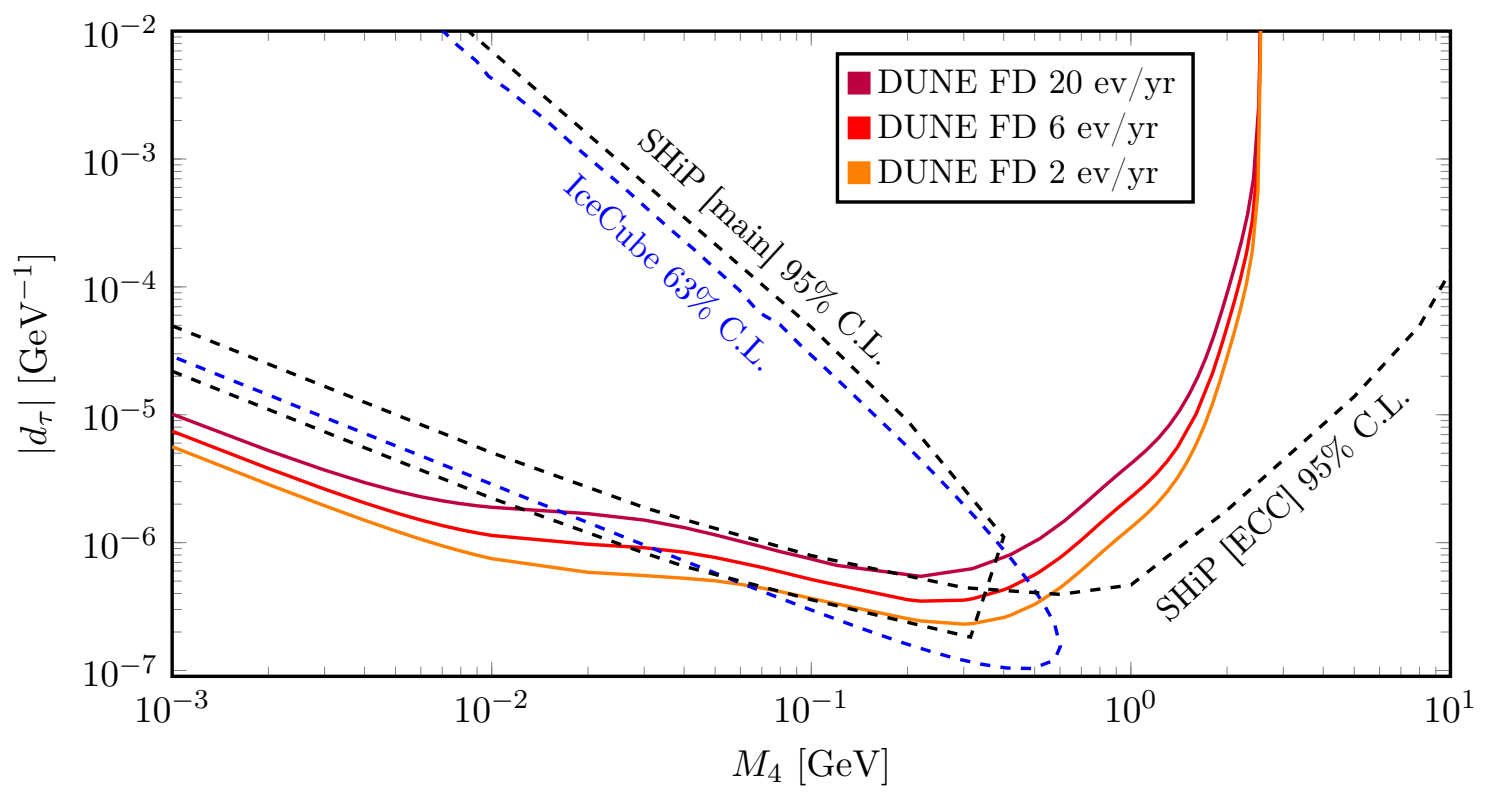

Figure 4. 2-, 6-, 20-events/yr curves at the DUNE far detector (orange, red, purple, respectively). For comparison we show the $95 \%$ C.L. sensitivities at SHiP from [1] assuming 100 background events over 5 years of exposure, equivalently $2 \times 10^{20} \mathrm{POT}$, (dashed black) for two detector types (ECC and main detector). In dashed blue is the one double-bang event/6 yrs curve at IceCube from [7].

by atmospheric neutrinos and decaying via the dipole operator [7]. The authors calculate the curve for 1 event/ 6 years, corresponding to an exclusion of $1-e^{-1} \approx 63 \%$ assuming no background. We note that the original curve used $\mu_{\mathrm{tr}} \equiv 2 d$ and units of $\mu_{B} \approx 296.1 \mathrm{GeV}^{-1}$.

\subsection{Near-detector sensitivity to the $\nu_{e}$ and $\nu_{\mu}$ dipole portal}

It is possible to use the intense muon-, and somewhat-less-intense electron-neutrino fluxes to constrain $d_{e}$ and $d_{\mu}$ at the near detector. We consider a signal from up-scattering to the HNL in close analogy to the FD signal described in section 2. Our method used earlier to calculate the event rates at the far detector (for both inside and outside events) can be applied to this situation with the following modifications: the baseline becomes $L_{\mathrm{ND}}=574 \mathrm{~m}$, the oscillation probability is set to one, and we use the intrinsic muonand electron-neutrino fluxes from [37]. We consider only the ND on-axis configuration and assume the detector fiducial volume is $6 \mathrm{~m}$ wide, $2 \mathrm{~m}$ high and $3 \mathrm{~m}$ long with a fiducial mass of $50 \mathrm{t}$ [41, section 2.7]. The crust at the surface of the Earth has a density of $2.6 \mathrm{~g} / \mathrm{cm}^{3}$ [38], whereas soil has a density 1.1 to $1.6 \mathrm{~g} / \mathrm{cm}^{3}$ [42]; we take an average density of $2 \mathrm{~g} / \mathrm{cm}^{3}$ and we exclude the region inside and next to the decay pipe $r_{p} \geq 270 \mathrm{~m}$ (see [36, figure 1.2]); but we still assume a point-like neutrino source. (This is conservative, since decays along the pipe will only lessen the $1 / r_{p}^{2}$ geometric suppression.) The near detector has a depth of $62 \mathrm{~m}$, so the angle of the beam with respect to ground level is $\operatorname{arcsine}(62 \mathrm{~m} / 574 \mathrm{~m}) \approx$ $108 \mathrm{mrad}>\theta_{b}^{\max } \equiv 62.72 \mathrm{mrad}$, so we can safely set $\varepsilon_{\varphi_{b}}$ to one. 
Our results for the $d_{e}$ and $d_{\mu}$ transition moments are shown below in figures 5 and 6 , respectively, in comparison with current limits as well as future sensitivities (see section 3.3 for further discussion). We find that the DUNE-ND six-events/year curves cover unconstrained parameter space in both cases and are competitive with various prospective sensitivities.

Some comments are in order. Our analysis takes into account only HNL production via the up-scattering process. As discussed in [1] there will also be direct production from meson decays produced in the target, either mediated by a virtual neutrino or photon: $P^{ \pm} \rightarrow \ell^{ \pm} \stackrel{(-)}{\nu} \gamma, P^{0} \rightarrow \gamma \nu_{\alpha} \nu_{4}$, where $P$ denotes a pseudo-scalar meson and $\ell$ a charged lepton. If the HNL is long-lived enough to reach the detector, such processes will give an additional contribution to the signal. The calculation of these events would involve a detailed consideration of meson production in the beam target, which is beyond the scope of this work. Note that these processes will give only an additional contribution to the signal; therefore our sensitivities are conservative in this respect. However, due to the large $\nu_{\mu}$ flux at the ND, one expects also significantly more SM NC events than in the FD, leading to a larger background from $\mathrm{NC} \pi^{0}$ events.

In principle one may expect also some prompt flux of tau neutrinos produced at the beam target from heavy-meson decays. This could be used, for instance, in the proposed SHiP experiment to search for HNLs mixed with $\nu_{\tau}[14,43]$ and has been considered in [1] to calculate the SHiP sensitivity to the dipole portal $d_{\tau}$. At DUNE the beam energy is lower than at $\mathrm{SHiP}$ and one expects a much reduced $\nu_{\tau}$ flux. Indeed, there is no tau-neutrino flux available in the flux files provided by DUNE [37]. The $\nu_{\tau}$ flux generated at the DUNE beam has been estimated recently in [22-24]. In principle this flux could be used also to constrain $d_{\tau}$ at the DUNE ND. Extrapolating the results from [23] we have estimated, however, that the signal from the direct $\nu_{\tau}$ flux in the near detector would be subleading to the one due to $\nu_{\mu} \rightarrow \nu_{\tau}$ oscillations in the far detector considered in the present work (see appendix D). Therefore we concentrate here on the FD for $d_{\tau}$.

On the other hand, the $\nu_{\mu}$ and $\nu_{e}$ fluxes (after taking into account oscillations) will also produce a HNL signal from $d_{\mu}$ and $d_{e}$ in the FD; this signal for $d_{\mu}$ should be comparable in strength to the one for $d_{\tau}$ and somewhat weaker for $d_{e}$. Therefore, comparing the ND sensitivity curves from figures 5 and 6 to the one from the FD in figure 7 , we expect the ND to provide superior sensitivity to $d_{e}$ and $d_{\mu}$, due to much larger fluxes at the ND.

\subsection{Global picture}

Let us now summarise various constraints and sensitivities on the HNL dipole portal and set our results for the DUNE FD and ND in the global context. In figures 5 to 7 we show the landscape of current constraints and projected sensitivities of $d_{\alpha}$ versus $M_{4} \cdot{ }^{3}$ Constraints from previous experiments are shaded with solid boundaries and sensitivities based on future experiments and estimated exclusions (for which there is no rigorous background/selection-efficiency analysis) are shown with dashed lines except for the DUNE

\footnotetext{
${ }^{3}$ The magnetic moments of SM neutrinos may also lead to observable corrections in all kinds of neutrino experiments and some astronomical and cosmological processes. The relevant discussions and stringent limits can be found in $[44,45]$.
} 


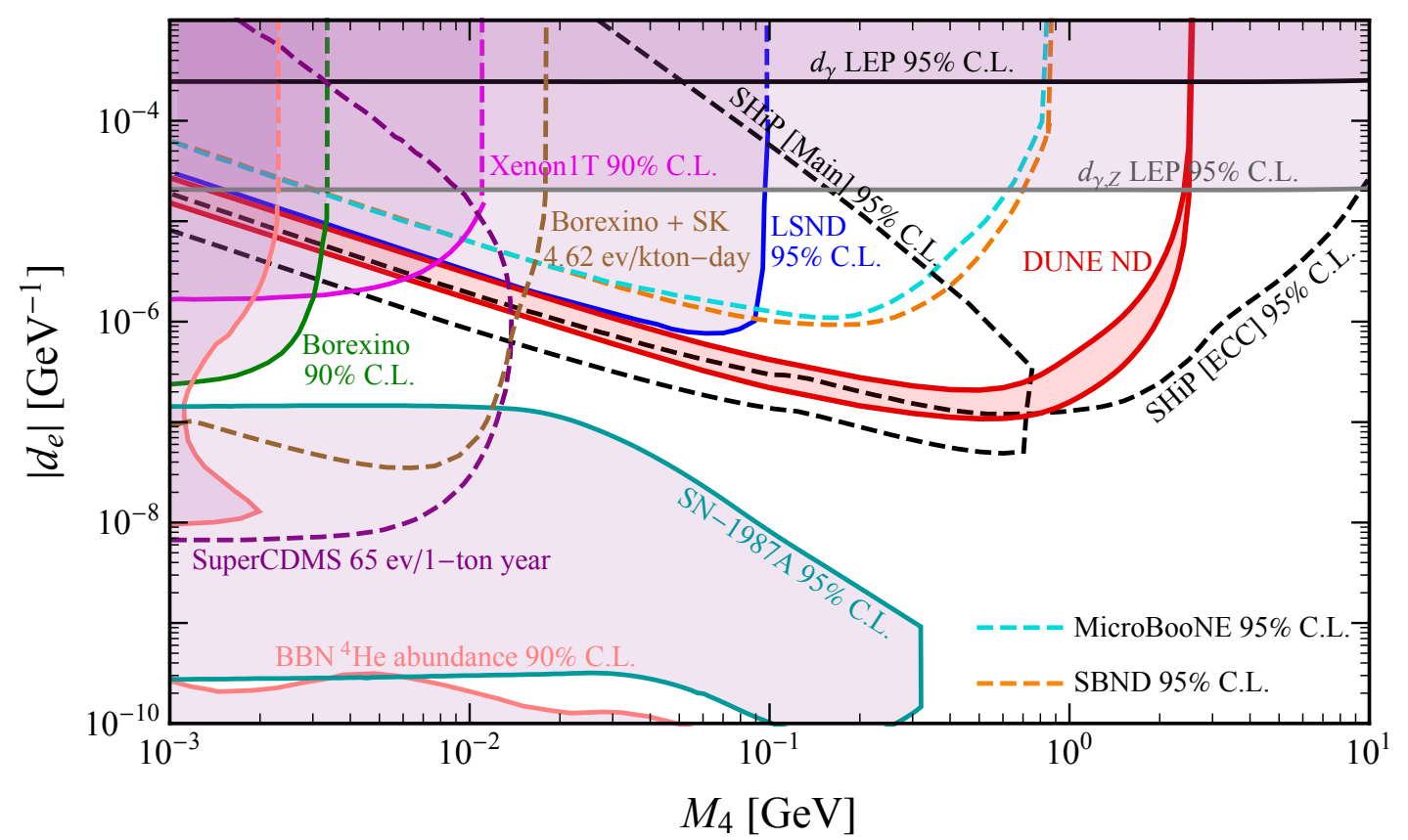

Figure 5. Comparison of the DUNE-ND sensitivity to $\left|d_{e}\right|$ obtained in this work (red solid) with current constraints (solid curves, shaded regions), and sensitivities of future projects or estimated exclusions (dashed curves). The band represents the region with 2-20 events/year, corresponding to $95 \%$ C.L. sensitivity over 5 years with $25-2500$ background events. Limits and sensitivities are from LSND, MicroBooNE, SBND, SHiP, LEP, SN 1987A [1], solar neutrinos [3, 6], Xenon1T, BBN ${ }^{4}$ He abundance [3], SuperCDMS [4].

ND and FD (being highlighted in solid lines), the results of this work. In order to illustrate the impact of background events, our results are presented as a band, showing the region with 2 to 20 events/year. Using signal/ $\sqrt{\text { background }} \approx 2$ this would correspond to a $95 \%$ C.L. sensitivity over 5 years for the range of 25 to 2500 background events. As discussed above, background and efficiency considerations will be rather different for the different classes of events (outside/inside coherent/inside incoherent), a subtlety which we ignore here. A detailed study along these lines is beyond the scope of this work; nevertheless the bands shown in the figures of this section gives a rough indication of the potential impact of background events or selection efficiencies.

We summarise below the various constraints and projected sensitivities in figures 5 to 7 :

- We show the dominant constraints from LSND and MiniBooNE of $d_{e}$ and $d_{\mu}$, respectively [1]. The projected sensitivities to $d_{e}$ from SBND and MicroBooNE are also shown; for $d_{\mu}$ they are similar to MiniBooNE, and are not shown, see [1].

- By considering induced elastic scattering of $\nu_{\mu}\left(\bar{\nu}_{\mu}\right)$ on electrons, CHARM-II can constrain $d_{\mu}$ (dark yellow) $[7,46]$. In a similar fashion, DONUT (an accelerator experiment dedicated to investigate tau-neutrino interactions) gave an upper limit on $d_{\tau}$ [48], which applies for $M_{4} \lesssim 0.3 \mathrm{GeV}$ (due to kinematics). 


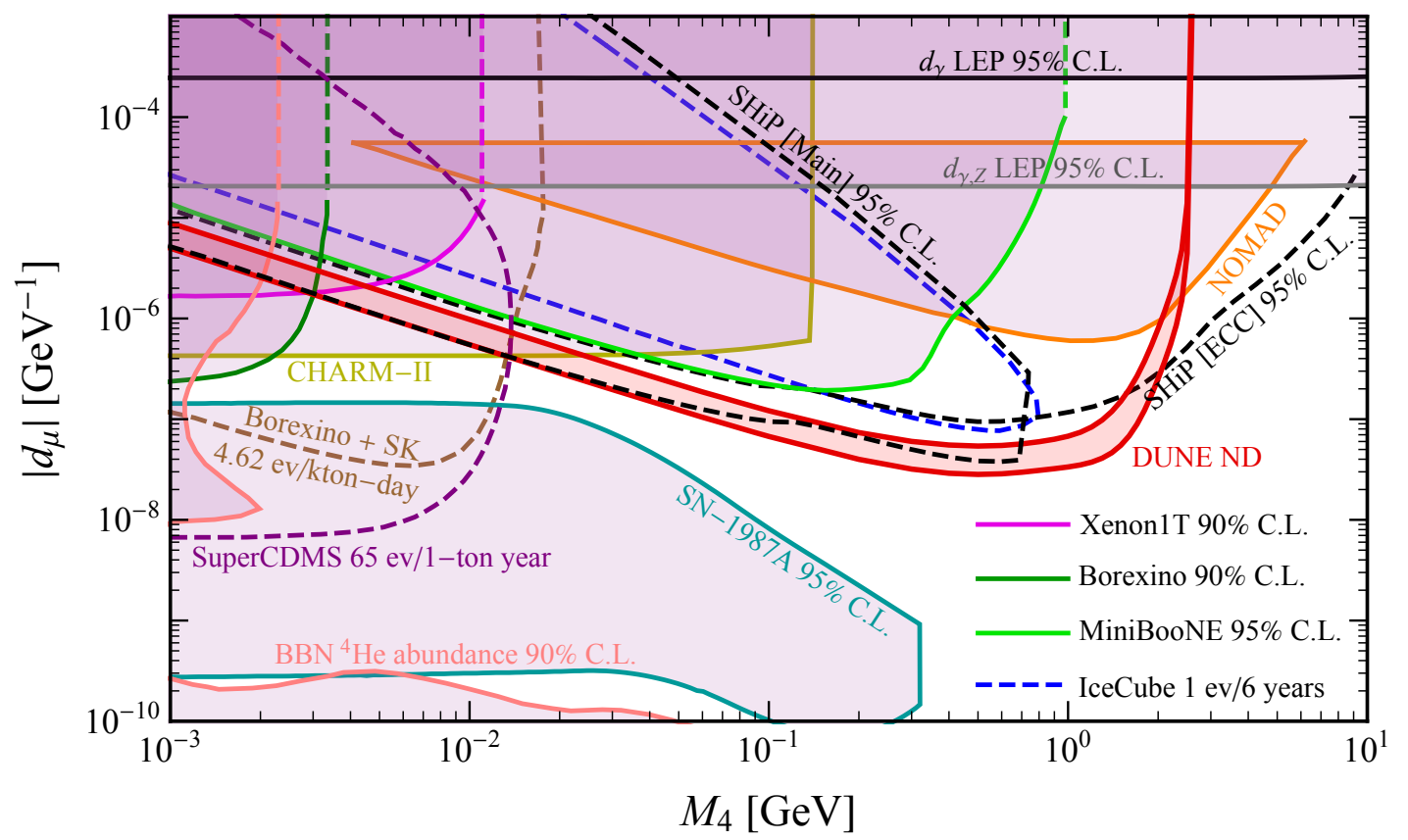

Figure 6. Comparison of the DUNE-ND sensitivity to $\left|d_{\mu}\right|$ obtained in this work (red solid) with current constraints (solid curves, shaded regions), and sensitivities of future projects or estimated exclusions (dashed curves). The band represents the region with 2-20 events/year, corresponding to $95 \%$ C.L. sensitivity over 5 years with $25-2500$ background events. Limits and sensitivities are from CHARM-II [7, 46], NOMAD [1, 47], Icecube [7], solar neutrinos [3, 6], MiniBooNE, SHiP, LEP, SN 1987A [1], Xenon1T, BBN ${ }^{4} \mathrm{He}$ abundance [3], SuperCDMS [4].

- At the SPS at CERN, the past NOMAD experiment searched for the single-photon signal from the up-scattering of neutrinos into sterile neutrinos (constraint from [1, 47] in orange in figure 6). The proposed SHiP detector [49] (also at the SPS) consists of an emulsion cloud chamber (ECC) near detector and a main detector. Sensitivities from [1] are shown as black dashed curves. For electron and tau flavours a background of 100 events is assumed, while for the muon flavour a background level of 1000 events is assumed.

- We also show bounds calculated in [1] from LEP; however these depend on the UVcompletion of the model, as above the electroweak scale the dipole operator must couple to the fields before electroweak symmetry breaking, which allows on-shell $Z$ or $W$ production. The solid black curves ignore on-shell $W$ and $Z$ production, while the solid grey include on-shell $Z$ s. (See also table II and figure 9 of [1].)

- Constraints from modifications of the solar-neutrino electronic recoil spectrum at Borexino due to transition magnetic moments $[50,51]$ were calculated for the HNL portal in [3] and are shown in dark-green in figures 5 to 7. An analysis of solarneutrino nuclear recoils from the Xenon1T dark-matter experiment leads to the constraints on $d_{\alpha}$ shown in magenta [3]. Constraints from the same type of signal at 


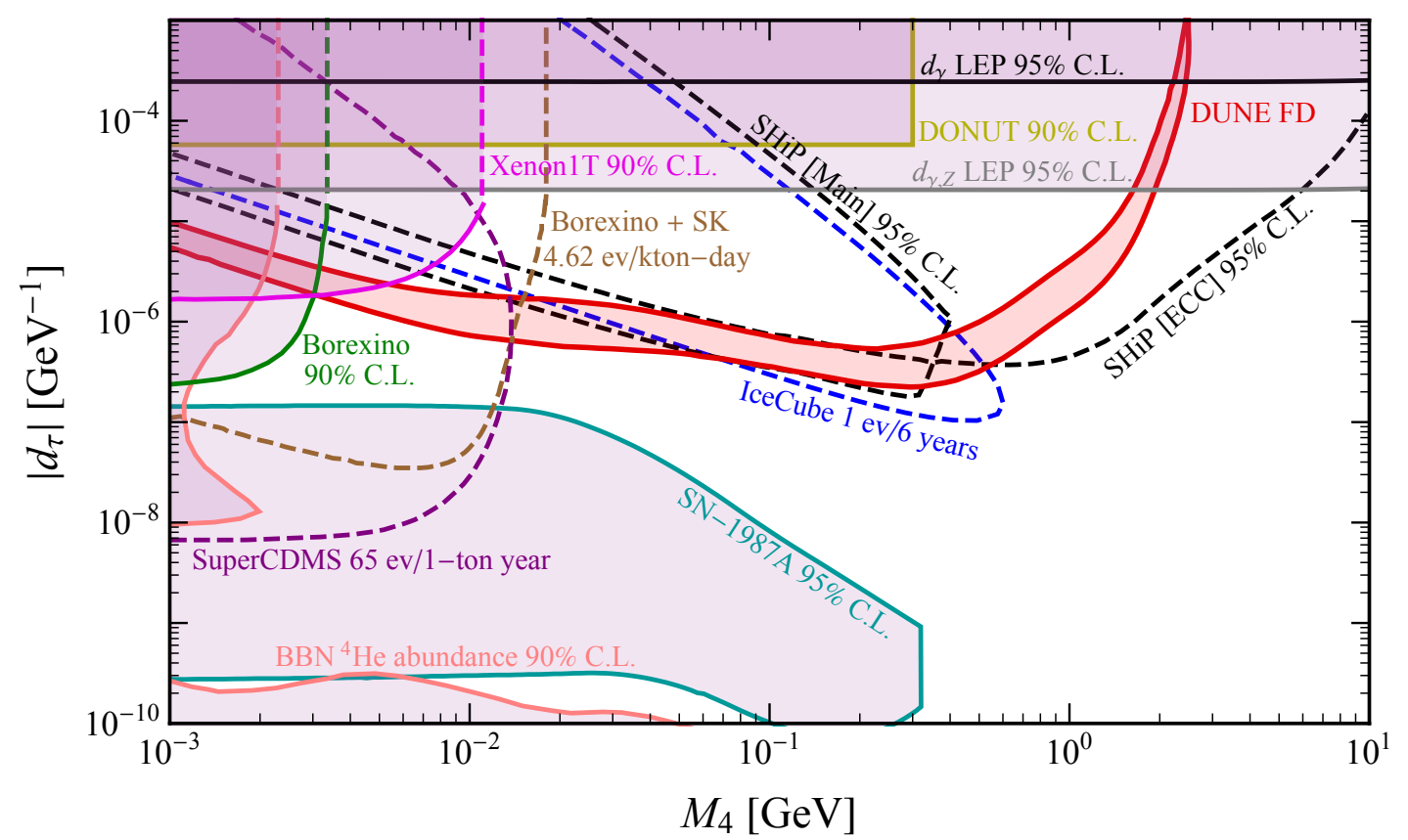

Figure 7. Comparison of the DUNE-FD sensitivity to $\left|d_{\tau}\right|$ obtained in this work (red solid) with current constraints (solid curves, shaded regions), and sensitivities of future projects or estimated exclusions (dashed curves). The band represents the region with 2-20 events/year, corresponding to $95 \%$ C.L. sensitivity over 5 years with 25-2500 background events. Limits and sensitivities are from DONUT [48], Icecube [7], solar neutrinos [3, 6], SHiP, LEP, SN 1987A [1], Xenon1T, BBN ${ }^{4}$ He abundance [3], SuperCDMS [4].

the future SuperCDMS dark matter detector are shown in purple assuming a 1-ton year exposure time and $3 \sigma$-significance (65 events) [4]. Due to oscillations, there is an $\mathcal{O}(1)$ correction to the $d_{e}$ constraints.

- A recent analysis [6] considers a situation, similar to ours, of up-scattering of solar neutrinos in the Earth and decay in the detector; it combines data from both Borexino [52] and Super-Kamiokande (SK) [53] to derive the constraints of $d_{\alpha}$ (brown, dashed); however this analysis does not consider the geometric suppression rigorously for outside events.

- From the observed SN 1987A neutrino burst limits on $d_{\alpha}$ can be set [1]. The areas enclosed by the cyan curves in figures 5 to 7 are disfavoured by SN 1987A. Below the curve, the induced cooling effect is too weak, and above the interaction becomes strong enough so that steriles cannot escape the collapsing core. Finally, if the sterile is too heavy, the gravitational pull will also prevent it from leaving the supernova, leading to the vertical cut-off of the exclusion curve.

- In [7], bounds from double-bang events (a signal with two visibly separate cascades) at IceCube from the atmospheric neutrino flux were calculated. These are denoted 
by the blue dashed lines in figures 6 and 7, namely one event during a data-taking period of six years.

- Bounds from cosmology and Big-Bang Nucleosynthesis (BBN) are shown in pink. The dipole interaction alters the expansion and cooling rates of the universe, leading to a corrected neutron-to-proton ratio and baryon-to-photon ratio [3]. The final ${ }^{4} \mathrm{He}$ abundance depends on $M_{4}$ and $d_{\alpha}$. For the observed primordial- ${ }^{4} \mathrm{He}$ fraction $Y_{p}=$ $0.245 \pm 0.006(2 \sigma)$, the corresponding constraints of $d_{\alpha}$ are illustrated in figures 5 to 7 .

\section{DUNE-FD sensitivity to active-sterile mixing via $U_{\tau 4}$}

So far we assumed that the mixing of the sterile neutrino with active flavours is negligible compared to the dipole interaction. In this section we briefly comment on the opposite case when the heavy neutrino mixes with strength $\left|U_{\alpha 4}\right|^{2}$ with active neutrinos and the dipole interaction is negligible. There are strong bounds on mixing with electron and muon flavour, as well as excellent prospects for upcoming projects, see for example [11, 22-24, 43, 54, 55]. Therefore, we focus on mixing with the tau flavour $\left|U_{\tau 4}\right|^{2}$, which is more difficult to probe.

We explore a similar phenomenology as in the case of the dipole: we use the $\nu_{\tau}$ flux generated by $\nu_{\mu} \rightarrow \nu_{\tau}$ oscillations in the DUNE experiment. ${ }^{4}$ These neutrinos can interact via $\mathrm{NC}$ interactions in the Earth or inside the far detector and up-scatter to $\nu_{4}$. Instead of the massless photon mediator, up-scattering is mediated by $Z^{0}$-exchange. As previously mentioned, since the mediator is massive, the total cross section depends linearly on the target mass (rather than logarithmically as in the dipole case) and therefore up-scattering on electrons can be neglected. Subsequently the heavy neutrino can decay and leave an observable signal in the detector. The main decay processes for $M_{4}<1 \mathrm{GeV}$ and mixing only with the tau flavour are $\nu_{4} \rightarrow \nu_{\tau} \nu_{l} \bar{\nu}_{l}, \nu_{4} \rightarrow \nu_{\tau} e^{+} e^{-}$and $\nu_{4} \rightarrow \nu_{\tau} \pi^{0}$, the latter two providing a visible signal. There is considerable confusion in the literature about the decay widths of a sterile neutrino that mixes with SM neutrinos with disagreeing results, compare e.g. $[11,23,54,55,58]$. In our work we use the formulae presented recently in [23] where a discussion on various previous results can also be found.

In figure 8 we show the curves in the plane of heavy-neutrino mass $M_{4}$ and mixing parameter $\left|U_{\tau 4}\right|^{2}$ corresponding to 1 signal event/year in the DUNE FD separately for inside and outside events. We see that the sensitivity covers a small region at relatively large mixing between the current exclusion limits from DELPHI $[54,59]$ and CHARM [58]. Let us note that a disagreement in the decay rate for $\nu_{4} \rightarrow \pi^{0} \nu_{\tau}$ used in [58] compared to recent papers (e.g. $[23,55]$ ) may potentially effect the CHARM bound shown in the figure.

Also shown in the figure is the sensitivity of the DUNE near detector from [23] (90\% C.L. sensitivity over 7 years of running, or $7.7 \times 10^{21} \mathrm{POT}$ ). This signal comes from the (small - though non-zero) sterile-neutrino flux produced by meson decays in the target to probe heavy-neutrino mixing with the tau flavour. The authors assumed a $20 \%$ selection efficiency with no background (corresponding to 2.44 events over 7 years for

\footnotetext{
${ }^{4}$ For similar considerations in the context of solar and atmospheric neutrinos see [56, 57].
} 


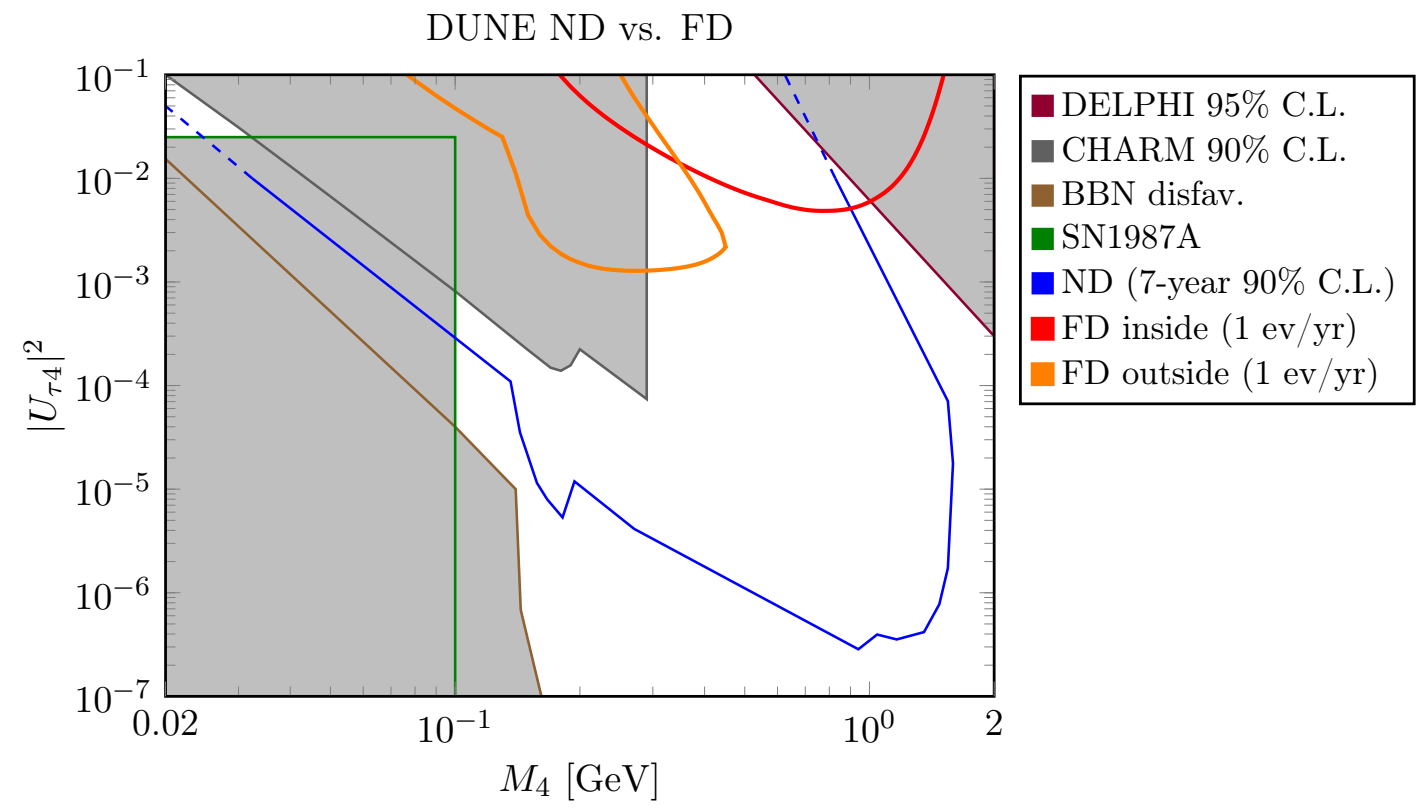

Figure 8. Comparison of the one-event/yr curves (100\% efficiency) for inside and outside events at the DUNE FD with the 7-year 90\% C.L. sensitivity at the DUNE ND assuming a $20 \%$ selection efficiency and no background from [23]. The ND curve has been extrapolated to higher mixings (dotted line). We also show the existing limits from DELPHI [54, 59] and CHARM [58] as well as BBN and SN 1987A constraints from [60].

90\% C.L. sensitivity). ${ }^{5}$ Assuming the same selection efficiency, our one-event curve corresponds to $7 \times 20 \%=1.4$ events over 7 years. As obvious from this figure, for the case of mixing the FD cannot compete with the ND. The difference with the dipole case is that the event rate in the FD relative to the ND is suppressed by the NC cross section involved in the up-scattering. A summary of the sensitivity to this scenario of various other planned projects (including SHiP) can be found e.g. in [24].

BBN constraints from [60] are also shown in figure 8, corresponding to $\Delta N_{\text {eff }}=0.2$. For masses $M_{4}$ larger than the neutral-pion mass, the bound weakens due to the large visible decay width. From ibid. bounds are also derived from the SN 1987A observation based on the thesis that any emission into exotic dark sectors would reduce the duration of the neutrino burst, which is $\sim \mathcal{O}(1 \mathrm{~s})$ (see also [61, 62]). The bound is $\left|U_{\tau 4}\right|^{2} \lesssim 7.5 \times 10^{-9}$ for $M_{4} \lesssim 100 \mathrm{MeV}$; but if the sterile neutrino's decay length is too short, it never leaves the collapsing star's core and doesn't contribute to the energy loss. The authors estimate this to occur when $\left|U_{\tau 4}\right|^{2} \gtrsim 2.5 \times 10^{-2}$, where we cut off the bound.

\footnotetext{
${ }^{5}$ Recently a similar study has been performed in [24] including more realistic detector simulations and background considerations. In [22] the sensitivity to HNL mixing (including the tau flavour) of a so-called "multi-purpose" near detector at DUNE has been considered.
} 


\section{Conclusion}

We have calculated the sensitivity of the DUNE experiment to the dipole portal with a heavy neutral lepton. Given the neutrino beam energy, DUNE can probe HNLs in the mass range from a few $\mathrm{MeV}$ to a few $\mathrm{GeV}$, with a peak sensitivity for masses $M_{4} \simeq 300$ to $500 \mathrm{MeV}$. We focus here on HNL production via the up-scattering of active neutrinos due to photon-mediated interactions with the matter surrounding the detector or inside the detector (for sufficiently short-lived sterile neutrinos). The signal consists of single-photon events either with or without a displaced NC-type interaction (depending on whether the up-scattering happens inside or outside the detector and whether the scattering happens coherently on the full nucleus).

We have shown that the far detector provides a competitive limit for the transition magnetic moment between the tau neutrino and the HNL, thanks to the sizeable tau neutrino flux generated by $\nu_{\mu} \rightarrow \nu_{\tau}$ oscillations. DUNE will be able to probe the tauneutrino dipole portal down to $d_{\tau}$ of order a few $\times 10^{-7} \mathrm{GeV}^{-1}$. Electron- and muonneutrino dipole portals are more efficiently probed at the near detector, thanks to the large flux of these flavours. Restricting our analysis to up-scattering-induced events, we find sensitivities for $d_{e}$ down to $10^{-7} \mathrm{GeV}^{-1}$ and a few $\times 10^{-8} \mathrm{GeV}^{-1}$ for $d_{\mu}$. Our results for DUNE are compared in section 3.3 to various present constraints and upcoming sensitivities available in the literature.

Motivated by these results, one may ask the question, whether the oscillation-induced flux of tau neutrinos at the far detector can also be used to test HNL mixing with $\nu_{\tau}$. While we find indeed that the far detector can test a currently unconstrained region, it turns out that in this case the signal in the near detector from the prompt HNL flux dominates. The reason is the additional suppression of the far detector flux due to the $\mathrm{NC}$ cross section required for the $\nu_{\tau} \rightarrow$ HNL up-scattering.

As an outlook, let us mention some topics for future work. First, we concentrate here on estimating the size of the signal. A reliable sensitivity calculation needs to take into account a detailed background analysis, and making use of event discrimination abilities in the liquid argon detector. Second, we have focused here on the HNL production via the up-scattering of active neutrinos mediated by the magnetic-moment interaction. In principle, the same interaction will also lead to HNL production in the beam target from meson decays via virtual-neutrino or -photon exchange. These HNLs may contribute to the signal in the near detector. Predicting such a signal requires a detailed simulation of the meson production and decay in the beam target and decay pipe, which is beyond the scope of the present work.

To conclude, a transition magnetic moment $d_{\alpha}$ between active and sterile neutrinos provides an attractive portal to search for physics beyond the Standard Model. We have shown that the DUNE experiment in its standard configuration has promising potential to test all three flavours, $d_{e, \mu, \tau}$, when considering both the near and far detectors. Our results warrant more detailed studies in terms of background estimates and signal discrimination methods. 


\section{Acknowledgments}

We thank Mahmoud Al-Awashra for collaboration at the initial stages of this project and Ryan Plestid for useful discussions. Albert Zhou thanks the Doctoral School "Karlsruhe School of Elementary and Astroparticle Physics: Science and Technology (KSETA)" for financial support through the GSSP program of the German Academic Exchange Service (DAAD). This project has received support from the European Union's Horizon 2020 research and innovation programme under the Marie Sklodowska-Curie grant agreement No 860881-HIDDeN. Jing-yu Zhu is supported partly by the China and Germany Postdoctoral Exchange Program from the Office of China Postdoctoral Council and the Helmholtz Centre (Grant No. 2020031) and by the National Natural Science Foundation of China (Grant No. 11835005 and 11947227).

Note added. After the completion of this work the preprint [63] appeared, with partially similar considerations. Those authors consider what they call double-bang events, when both the hadronic and single-photon decay signal are visible. These would be a subclass of events considered here. Comparing with their DUNE analysis, where our results overlap we find good agreement.

\section{A Cross sections}

We summarise here the relevant scattering cross sections $[1,3]$ :

$$
\begin{aligned}
\frac{\mathrm{d} \sigma\left(\nu_{\tau} e^{-} \rightarrow \nu_{4} e^{-}\right)}{\mathrm{d} Q^{2}}= & \alpha_{\mathrm{QED}}\left(\frac{\left|d_{\alpha}\right|}{\mathrm{GeV}^{-1}}\right)^{2}\left[\frac{2}{Q^{2}}-\frac{1}{m_{e} E_{\nu}}+\frac{M_{4}^{2}}{2 m_{e} Q^{2} E_{\nu}^{2}} \times\right. \\
& \left.\left(E_{r}-m_{e}-2 E_{\nu}+M_{4}^{2} \frac{E_{r}-m_{e}}{Q^{2}}\right)\right] \times 3.894 \times 10^{-28} \mathrm{~cm}^{2} / \text { nucleon } \\
\equiv & X\left(m_{e}\right) ; \\
\frac{\mathrm{d} \sigma_{\text {nucleon }}}{\mathrm{d} Q^{2}}= & \frac{\mathrm{d} \sigma\left(\nu_{\tau} p \rightarrow \nu_{4} p\right)}{\mathrm{d} Q^{2}}+\frac{\mathrm{d} \sigma\left(\nu_{\tau} n \rightarrow \nu_{4} n\right)}{\mathrm{d} Q^{2}} ; \\
\frac{\mathrm{d} \sigma\left(\nu_{\tau} p / n \rightarrow \nu_{4} p / n\right)}{\mathrm{d} Q^{2}}= & F_{1, p / n}^{2} X\left(m_{p / n}\right)+\alpha_{\mathrm{QED}}\left(\frac{\left|d_{\alpha}\right|}{\mathrm{GeV}^{-1}}\right)^{2} \mu_{\mathrm{N}}^{2} \times \\
& \left(\frac{F_{2}^{p / n}}{E_{\nu}}\right)^{2}\left[2\left(2 E_{\nu}-E_{r}\right)^{2}-2 Q^{2}+\frac{M_{4}^{2}}{m_{p / n}}\left(E_{r}-4 E_{\nu}+M_{4}^{2} / E_{r}\right)\right] \\
\frac{\mathrm{d} \sigma\left(\nu_{\tau} N \rightarrow \nu_{4} N\right)}{\mathrm{d} Q^{2}}= & \frac{2 Z^{2}}{A} F_{\text {nucleus }}^{2} X\left(M_{N}\right)
\end{aligned}
$$

where $E_{r} \equiv \frac{Q^{2}}{2 M_{T}}$ is the recoil energy and $\mu_{\mathrm{N}} \equiv \frac{e}{2 m_{p}} \approx 0.16 / \mathrm{GeV}$ is the nuclear magneton. The numerical constant arises from the conversion from $\mathrm{GeV}^{-2}$ to $\mathrm{cm}^{2}$. Note that we normalise cross sections always per nucleon, not nucleus. This implies that the incoherent 
cross sections are a factor 2 less than naïvely expected, since on average there are twice as many nucleons than protons, neutrons and electrons. We also note that compared to other publications, we use $d \equiv 2 \mu_{\mathrm{tr}}$. The relevant form-factors are

$$
\begin{aligned}
F_{1}^{p} & =\left(1+\frac{\eta}{1+\eta} a_{p}\right) G_{D}, & F_{1}^{n} & =\frac{\eta}{1+\eta} a_{n} G_{D} \\
F_{2}^{p / n} & =\frac{a_{p / n}}{1+\eta} G_{D}, & G_{D} & =\left(1+\frac{Q^{2}}{0.71 \mathrm{GeV}^{2}}\right)^{-2} \\
a_{p} & =\mu_{p}-1 & a_{n} & =\mu_{n}, \quad \eta \equiv \frac{Q^{2}}{4 M_{T}^{2}} .
\end{aligned}
$$

for the nucleon, where $a_{p / n}$ the anomalous magnetic moments of the proton/neutron: 1.793 and -1.913 , respectively; and finally

$$
\begin{aligned}
F_{\text {nucleus }} & =\frac{3 j_{1}\left(Q R_{\text {nucleus }}\right)}{Q R_{\text {nucleus }}} \exp \left[-\frac{(Q s)^{2}}{2}\right] \\
R_{\text {nucleus }} & =\sqrt{(1.23 \sqrt[3]{A}-0.6)^{2}+\frac{7}{3}(\pi a)^{2}-5 s^{2}} \mathrm{fm} \\
s & =0.9 \mathrm{fm} \quad a=0.52 \mathrm{fm}
\end{aligned}
$$

for the nucleus [64, section 4].

Kinematic relations. For the inside events, the spectrum is evaluated as a function of $E_{\nu}$. In order to convert from $\mathrm{d} / \mathrm{d} Q^{2}$ to $\mathrm{d} / \mathrm{d} \cos \theta_{s}$ we use kinematics to obtain

$$
\left(E_{\nu}+M_{T}\right) E_{4}-\left(M_{T} E_{\nu}+M_{4}^{2} / 2\right)=E_{\nu} p_{4} \cos \theta_{s} .
$$

Define the following relations

$$
\begin{array}{rlrl}
A_{ \pm} & =M_{T}+E_{\nu}\left(1 \pm \cos \theta_{s}\right), & B=M_{T} E_{\nu}+M_{4}^{2} / 2, \\
\Delta & =1-\left(M_{4} / B\right)^{2} A_{+} A_{-}, & C=\sqrt{\Delta}, \\
E_{4}^{0} & =B / A_{-},
\end{array}
$$

where $E_{4}^{0}$ corresponds to the heavy-neutrino energy in the limit $p_{4}=E_{4}$. The kinematic constraints are $M_{4}<E_{4}<E_{\nu}$ and $\Delta \geq 0$. This can be used to derive

$$
Q^{2}=2 M_{T}\left[\frac{E_{\nu}^{2}\left(1-\cos \theta_{s}\right)-M_{4}^{2} / 2}{A_{-}}+\frac{B(1-C)}{A_{+} A_{-}} E_{\nu} \cos \theta_{s}\right]
$$

and

$$
\frac{\mathrm{d} Q^{2}}{\mathrm{~d} \cos \theta_{s}}=-2 M_{T} \frac{\mathrm{d} E_{4}}{\mathrm{~d} \cos \theta_{s}}=\frac{2 M_{T} E_{\nu}}{A_{+}}\left[\frac{2 E_{4} E_{\nu} \cos \theta_{s}}{A_{-}}+E_{4}^{0} C+\frac{\left(M_{4} E_{\nu} \cos \theta_{s}\right)^{2}}{A_{-} B C}\right] .
$$

When $\Delta$ is very close to one, we expand in a Taylor series; the same goes for $p_{N}$ and other quantities with potentially small numbers. 
For the outside events, the spectrum is evaluated as a function of $E_{4}$. From eq. (A.7), one derives

$$
E_{\nu}=\frac{M_{T} E_{4}-M_{4}^{2} / 2}{M_{T}-E_{4}+p_{4} \cos \theta_{s}} .
$$

Then (from eq. (A.10)),

$$
\frac{\mathrm{d} \sigma}{\mathrm{d} \cos \theta_{s}} \frac{\mathrm{d} E_{\nu}}{\mathrm{d} E_{4}}=-2 M_{T} \frac{\mathrm{d} \sigma}{\mathrm{d} Q^{2}} \frac{\mathrm{d} E_{\nu}}{\mathrm{d} \cos \theta_{s}}=\frac{\mathrm{d} \sigma}{\mathrm{d} Q^{2}} \times \frac{2 M_{T} E_{\nu}^{2} p_{4}}{M_{T} E_{4}-M_{4}^{2} / 2} .
$$

\section{B Inside-event rate integral}

We provide here some details on the calculation of the integral to evaluate the inside-event rate. We focus on the far detector signal due to $d_{\tau}$. Modifications for the near detector signal are mentioned in section 3.2.

Neutrino flux. For inside events we can restrict to the on-axis flux, which we obtained from [37] in GLoBES format [65]. (These files are no longer available as of the writing of this article; please see [36, figure 5.4].) The flux is provided for the near detector, therefore a geometric suppression factor $L_{\mathrm{ND}}^{2} /\left|\vec{x}_{p}\right|^{2}$ was applied.

Oscillation probability. Matter effects change the $\nu_{\mu} \rightarrow \nu_{\tau}$ oscillation probability only at the $\mathcal{O}(1 \%)$-level. Therefore, we use the effective-two-flavour vacuum probability

$$
P_{\mathrm{osc}}=0.943 \cdot \sin ^{2}\left(\frac{\Delta m^{2}\left|\vec{x}_{p}\right|}{4 E_{\nu}}\right), \quad \text { where } \quad \Delta m^{2}=2.523 \times 10^{-3} \mathrm{eV}^{2} .
$$

Decay probability. The decay width in the sterile neutrino's rest-frame is

$$
\Gamma_{0}=\frac{\left|d_{\alpha}\right|^{2} M_{4}^{3}}{4 \pi}
$$

and the probability to decay inside the detector is

$$
P_{\mathrm{dec}}=1-\exp \left(-\gamma \ell_{d}\right)
$$

where $\gamma \equiv \Gamma_{0} M_{4} / p_{4}$ is the lab-frame decay rate and $\ell_{d}$ is the distance from the production point $\vec{x}_{p}$ to the edge of the detector in the direction $\vec{x}_{p}-\vec{x}_{d}$.

Reconstruction efficiency. The reconstruction efficiency as a function of photon momentum $\varepsilon\left(p_{\gamma}\right)$ was taken from [19, figure 4.26]. We assume that in the sterile neutrino's rest frame the distribution of photon momentum is isotropic. Boosting this into the labframe gives $p_{\gamma}\left(\theta_{0} ; p_{4}, M_{4}\right)$, where $\theta_{0}$ is the photon momentum's rest-frame polar angle. The efficiency, then, is

$$
\varepsilon\left(p_{4}, M_{4}\right)=\int_{0}^{2 \pi} \varepsilon\left[p_{\gamma}\left(\theta_{0} ; p_{4}, M_{4}\right)\right] \mathrm{d} \theta_{0} .
$$

Let us note that for a Dirac sterile neutrino, the differential decay width $\mathrm{d} \Gamma_{0} / \mathrm{d} \cos \theta_{d}$ is not isotropic in general $[20,21]$. The asymmetry depends on the relative complex phase of electric and magnetic dipole moments and, in particular, it vanishes if one of them is zero. We have checked, however, that even in the presence of an asymmetry the effect on the integral in eq. (B.4) is at the few percent level and has therefore negligible impact on our results. 


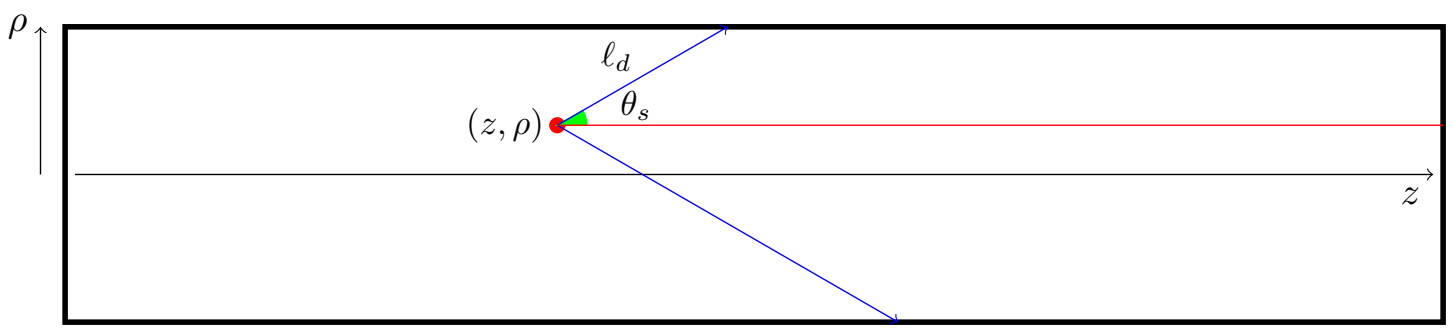

Figure 9. An incoming neutrino scatters at $(z, \rho)$ in the cylindrical detector module. In blue are two possible paths for the sterile neutrinos, at constant scattering angle $\theta_{s}$. In red is the trajectory of the neutrino, had it not scattered.

Approximations and geometry. Since the detector dimensions are $\mathcal{O}(10 \mathrm{~m})$, whereas the baseline is $L_{\mathrm{FD}} \approx 1300 \mathrm{~km}$ we can make the approximations $\left|\vec{x}_{p}\right| \approx L_{\mathrm{FD}}$ and ignore the beam opening angle on the scale of the detector size, as the maximum angle is $\theta_{b}=\mathcal{O}(10 \mathrm{~m}) / L_{\mathrm{FD}} \sim \mathcal{O}\left(10^{-5}\right) \approx 0$.

We assume that the detector is cylindrical with radius $r=\sqrt{12 \mathrm{~m} \cdot 14 \mathrm{~m} / \pi}$ and length $L_{d} \approx 58.2 \mathrm{~m}$. This allows us to do the $\varphi$ integral analytically. The geometry determining the decay length is sketched in figure 9 . In the figure, the decay path ends at the sides of the detector. There could be the case when both end at one of the ends of the detector. Then the decay-length is independent of the scattering position, defined by $\ell_{d}=\left(L_{d}-z\right) \sec \theta_{s}$ for the right end and $\ell_{d}=z \sec \theta_{s}$ for the left end. In this case our approximation $\ell_{d}=\ell_{d}(\rho=0) \equiv \ell_{d}^{0}$ is exact. We will not consider the case when the decay path ends partly on the side and partly at the end, as this will only occur rarely in comparison to the other two cases, and its effect will only be some kind of average between the two.

Let us consider the case, as drawn in the figure, when the decay path ends at the sides of the detector. From the figure it is clear that $\ell_{d} \sin \theta_{s}+\rho=r$. This is in the case $\varphi_{s}=0$. For non-zero $\varphi_{s}$,

$$
\left(\ell_{d} \sin \theta_{s} \cos \varphi_{s}+\rho\right)^{2}+\ell_{d}^{2} \sin ^{2} \theta_{s} \sin ^{2} \varphi_{s}=r^{2} .
$$

The solution to this quadratic equation (assuming $\theta_{s} \in[0, \pi]$ ) is

$$
\begin{aligned}
\ell_{d} & =\left[\sqrt{r^{2}-\rho^{2} \sin ^{2} \varphi_{s}}-\rho \cos \varphi_{s}\right] \operatorname{cosec} \theta_{s} \\
& =\ell_{d}^{0}\left[\sqrt{1-x^{2} \sin ^{2} \varphi_{s}}-x \cos \varphi_{s}\right], \quad x \equiv \frac{\rho}{r} .
\end{aligned}
$$

The penalty term (defined as the ratio of the exact decay probability to the $\rho=0$ approximation, $\left.P_{\text {dec }}\left(\ell_{d}\right) / P_{\text {decay }}\left(\ell_{d}^{0}\right)\right)$ can then be written as

$$
\begin{aligned}
\Pi\left(\gamma \ell_{d}^{0}\right) & =\left(1-\frac{2 \pi}{A_{\text {det. }}} \int_{0}^{r} \int_{0}^{2 \pi} e^{-\gamma \ell_{d}} \frac{\mathrm{d} \varphi_{s}}{2 \pi} \rho \mathrm{d} \rho\right) /\left(1-e^{-\gamma \ell_{d}^{0}}\right) \\
& =\left[1-\int_{0}^{1} \int_{0}^{2 \pi} \exp \left(-\gamma \ell_{d}^{0}\left\{\sqrt{1-x^{2} \sin ^{2} \varphi_{s}}-x \cos \varphi_{s}\right\}\right) \frac{\mathrm{d} \varphi_{s}}{\pi} x \mathrm{~d} x\right] / \\
& \left(1-e^{-\gamma \ell_{d}^{0}}\right) .
\end{aligned}
$$



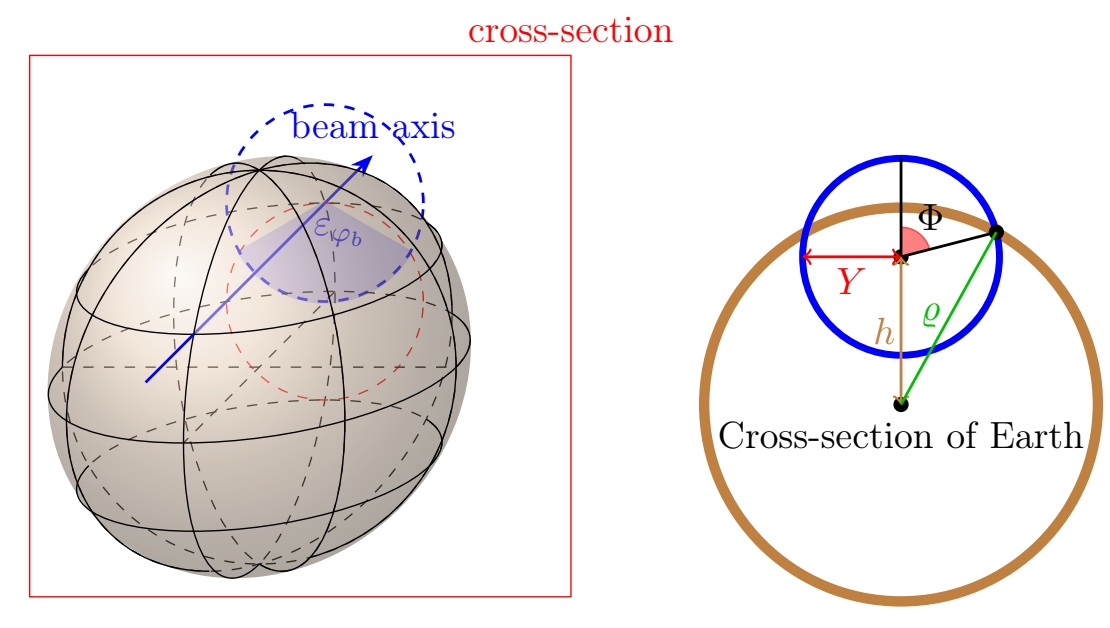

Figure 10. Example cross section with limits in $\varphi_{b}$. The function $\varepsilon_{\varphi_{b}}$ determines the relative contribution of the angles $\varphi_{b}$ inside the Earth.

Finally we must calculate $\ell_{d}^{0}$; the decay path ends at the sides of the detector if $l_{d} \cos \theta_{s} \leq L_{d}-z$ and $\theta_{s}<\pi / 2$, or $l_{d}\left|\cos \theta_{s}\right| \leq z$ and $\theta_{s} \geq \pi / 2$ (equivalently $l_{d} \cos \theta_{s}>-z$ ). Therefore,

$$
\ell_{d}^{0}=\left\{\begin{array}{lll}
\min \left[r \operatorname{cosec} \theta_{s},\left(L_{d}-z\right) \sec \theta_{s}\right] & \text { if } & \cos \theta_{s} \geq 0 \\
\min \left(r \operatorname{cosec} \theta_{s}, z\left|\sec \theta_{s}\right|\right) & \text { if } & \cos \theta_{s}<0 .
\end{array}\right.
$$

\section{Outside-event rate integral}

Here we provide some details for the outside-event rate calculations. Again we focus on the far detector signal due to $d_{\tau}$; modifications for the near detector signal are mentioned in section 3.2. Since evaluation of the cross section can be expensive, all terms in the integrand which depend purely on $\left(E_{\nu}, E_{4}\right)$ were pre-calculated and then stored in a bilinear interpolator. This table had to be recalculated for each mass $M_{4}$. The dependence on the decay width, which is non-linear, was also separated and recalculated for each $d_{\tau}$. The CQUAD GSL routine was used to undertake the integrals [66]. The $\mathrm{C}++$ source code can be provided upon request to Albert Zhou.

Off-axis flux. This case we take into account the different neutrino fluxes as a function of the off-axis angle. The off-axis (GLoBES) fluxes were obtained from [36, 37]. We take $62.72 \mathrm{mrad}$ as the maximum beam angle. Those files have units $\mathrm{GeV}^{-1} \mathrm{~m}^{-2} \mathrm{POT}^{-1}$. Bilinear interpolation is used to interpolate between neutrino energies and beam angles.

Evaluation of the $\varphi_{b}$-integral. In figure 10 (right), we see a cross section of the Earth, perpendicular to the beam-axis. Due to cylindrical symmetry, the integration occurs in a plane perpendicular to the radius of the Earth, with coordinates $(X, Y)=$ $\left(r_{p} \cos \theta_{b}, r_{p} \sin \theta_{b}\right)$. This plane is at a constant height $h=\sqrt{R_{\oplus}^{2}-\left(L_{\mathrm{FD}} / 2\right)^{2}}$ above the Earth (with the radius of the Earth $R_{\oplus}=6371 \mathrm{~km}$. In the perpendicular cross section, the rotation around $\varphi_{b}$ produces a circle of radius $Y$. The cross section shown is at a 
distance $\frac{L_{\mathrm{FD}}}{2}-X$ away from the actual centre of the Earth (the $X$-axis is perpendicular to the page). Thus, $\varrho=\sqrt{R_{\oplus}^{2}-\left(\frac{L_{\mathrm{FD}}}{2}-X\right)^{2}}$ and $\varepsilon_{\varphi_{b}} \equiv 2(\pi-\Phi)=2 \arccos \frac{Y^{2}+h^{2}-\varrho^{2}}{2 h Y}$. In the limit $Y=0$ however, we take $\varepsilon_{\varphi_{b}}=2 \pi$ for $X \in\left[0, L_{\mathrm{FD}}\right]$ and 0 otherwise. Arc-cosine is only well-defined in the interval $[-1,1]$. If its argument is $>1$, this means there is no intersection between the blue and brown circles, because the blue circle resides inside the brown: we set $\varepsilon_{\varphi_{b}}=2 \pi$; for the case $<-1$ the blue circle resides outside the brown one and $\varepsilon_{\varphi_{b}}=0$.

Decay probability, scattering solid angle and detector geometry. Let $\ell$ be the distance from production $\vec{x}_{p}$ to the detector $\ell=\sqrt{L_{\mathrm{FD}}^{2}+r_{p}^{2}-2 L_{\mathrm{FD}} r_{p} \cos \theta_{b}}$; then the decay probability is

$$
P_{\mathrm{dec}}=e^{-\gamma \ell}\left(1-e^{-\gamma L_{\mathrm{w}}}\right)
$$

where we have taken the detector width $L_{\mathrm{w}}=14 \mathrm{~m}$ as the characteristic detector dimension.

The scattering angle can be determined as

$$
\cos \theta_{s}=\frac{L_{\mathrm{FD}} \cos \theta_{b}-r_{p}}{\ell}
$$

this expression suffers from some numerical instability when the numerator has a larger magnitude than the denominator, in which case the fraction on the r.h.s. is set to its sign.

The scattering solid angle is

$$
\Delta \Omega_{s}=\sin \theta_{s} \Delta \theta_{s} \Delta \varphi_{s}=4 \arctan \frac{L_{d}}{2 \ell} \arctan \frac{L_{\mathrm{h}}}{2 \ell} \sin \theta_{s}
$$

where $L_{d}, L_{\mathrm{h}}$ are the length $58.2 \mathrm{~m}$ and height $12 \mathrm{~m}$ of the detector, respectively. In the limit of large $\ell$ (far away from the detector) and small $\gamma L_{\mathrm{w}}$,

$$
P_{\text {decay }} \cdot \Delta \Omega_{s}=e^{-\gamma \ell} \sin \theta_{s} \gamma L_{\mathrm{w}} L_{d} L_{\mathrm{h}} / \ell^{2} \propto V_{d} / \ell^{2}
$$

we see the signal is proportional to the detector volume and its angular size $\propto \ell^{-2}$, as expected. Away from this limit, the geometric dependence inside the angular size and decay probability will change how fast the signal decays; we neglect this affect. MonteCarlo studies with neutral-current up-scattering indicate that this effect results in a $\sim 20$ to $25 \%$ reduction of the spectrum (i.e. a penalty of 75 to $80 \%$ ). For the purpose of this study, we ignore this effect.

Oscillation phase. For small $E_{4}$, the spectrum $\mathrm{d} N / \mathrm{d} E_{4}$ has fast wiggles for low $E_{4}$. These arise from the oscillation probability. Assuming $E_{4} \sim E_{\nu}$, we set $P_{\text {osc }}$ to $1 / 2$ when

$$
E_{4}<\frac{\left(\Delta m^{2} / 4\right) L_{\mathrm{FD}}}{4 \pi}
$$

which is the fourth trough. The distance to the next trough is $\frac{\left(\Delta m^{2} / 4\right) L_{\mathrm{FD}}}{4 \cdot 5 \cdot \pi} \sim 6.6 \times 10^{-2} \mathrm{GeV}$. 
Integration boundaries. For masses $M_{4}>10^{-2} \mathrm{GeV}$ the integration boundaries are defined such that $\ell<10 / \gamma$, determined by the exponential decay of the decay probability; for lower masses this is not a good approximation. This results in

$$
\begin{aligned}
\hat{\ell} \equiv \frac{\ell_{\mathrm{max}}}{L_{\mathrm{FD}}} & =\frac{100}{L_{\mathrm{FD} \gamma}}, \quad \Delta_{G}=\sqrt{\hat{\ell}^{2}-\sin ^{2} \theta_{b}} ; \\
\frac{r_{\text {min. }}}{L_{\mathrm{FD}}} & = \begin{cases}0 & M_{4} \leq 10^{-2} \mathrm{GeV} \\
\max \left(0, \cos \theta_{b}-\Delta_{G}\right) & M_{4}>10^{-2} \mathrm{GeV},\end{cases} \\
\frac{r_{\text {max. }}}{L_{\mathrm{FD}}} & = \begin{cases}1.5 & M_{4} \leq 10^{-2} \mathrm{GeV} \\
\cos \theta_{b}+\Delta_{G} & M_{4}>10^{-2} \mathrm{GeV} .\end{cases}
\end{aligned}
$$

Furthermore, the detector itself is removed for $Y<15 \mathrm{~m},\left|X-L_{\mathrm{FD}}\right|<L_{d}$.

\section{Estimate of the near-detector signal}

In this appendix we provide a rough estimate of the signal induced in the near detector by the $d_{\tau}$ transition moment. Such a signal requires the presence of a prompt $\nu_{\tau}$ flux at the near detector. Such a flux is not available in the files provided by DUNE at [37]; however it has been estimated recently in $[23,24]$ to study the sensitivity of sterile neutrino mixing with the tau flavour. Here we use their results to get a rough estimate for the dipole signal in the ND.

In the limit of small mixing, the number of mixing-induced sterile neutrino decays, $N_{\text {mix }}$, can be estimated by

$$
N_{\text {mix }} \sim\left|U_{\tau 4}\right|^{2} \Gamma_{0} \frac{M_{4}}{p_{4}} \Delta \ell_{\text {det. }} N_{\tau} \varepsilon
$$

where $\Gamma_{0}$ is the rest-frame decay width and $N_{\tau}$ is the number of $\nu_{\tau}$ passing through the detector during a given time period. Using a typical momentum $p_{4} \sim 10 \mathrm{GeV}$ (see [23, figure 3]) and with the assumption that $\nu_{4} \rightarrow \pi^{0} \nu_{\tau}$ dominates (see [23, figure 2]) we have $\Gamma_{0}=\frac{G_{F}^{2} M_{4}^{3}}{32 \pi} f_{\pi}^{2}\left|U_{\tau 4}\right|^{2}\left[1-\left(M_{\pi^{0}} / M_{4}\right)^{2}\right]^{2}$. From figure 7 of [23] we can see that there are 2.44 events over 7 years at $M_{4}=0.2 \mathrm{GeV}$ and $\left|U_{\tau 4}\right|^{2}=10^{-5}$. Accounting for the $20 \%$ efficiency $\varepsilon$ and the assumed 7 years of exposure we obtain from eq. (D.1)

$$
N_{\tau} \Delta \ell_{\text {det. }} \sim 3.6 \times 10^{12} \mathrm{~m} / \mathrm{yr} .
$$

Moving now to the case of dipole interaction, the number of dipole decay events, $N_{\text {dip }}$, can be estimated as

$$
N_{\text {dip }} \sim \rho_{N}^{\mathrm{ND}} \sigma_{\text {tot }}\left(N_{\tau} \Delta \ell_{\text {det. }}\right),
$$

where we assume that all $\nu_{4}$ decay inside the detector. Using a near-detector mass of 50 tonnes, a volume of $3 \mathrm{~m} \times 5 \mathrm{~m} \times 7 \mathrm{~m}$ [41] we obtain a nucleon density of $2.9 \times 10^{23} \mathrm{~cm}^{-3}$. Taking for the dipole induced cross section $\sigma_{\text {tot }}$ from [1, eq. (3)] and $Z=18$ for Argon (we 
also divide by the number of nucleons $A=40$ ), we can estimate the signal for the dipole portal as

$$
N_{\text {dip }} \sim 1.8 \times 10^{-3}\left(\frac{d_{\tau}}{10^{-6} \mathrm{GeV}^{-1}}\right)^{2} \mathrm{yr}^{-1} \quad\left(M_{4}=0.2 \mathrm{GeV}\right) .
$$

Hence, this result suggests that the signal in the ND will only be marginally relevant compared to the FD signal. Our estimate applies to inside events. Outside events are expected to have the same order of magnitude.

Open Access. This article is distributed under the terms of the Creative Commons Attribution License (CC-BY 4.0), which permits any use, distribution and reproduction in any medium, provided the original author(s) and source are credited.

\section{References}

[1] G. Magill, R. Plestid, M. Pospelov and Y.-D. Tsai, Dipole Portal to Heavy Neutral Leptons, Phys. Rev. D 98 (2018) 115015 [arXiv:1803.03262] [INSPIRE].

[2] K.S. Babu, S. Jana and M. Lindner, Large Neutrino Magnetic Moments in the Light of Recent Experiments, JHEP 10 (2020) 040 [arXiv:2007.04291] [INSPIRE].

[3] V. Brdar, A. Greljo, J. Kopp and T. Opferkuch, The Neutrino Magnetic Moment Portal: Cosmology, Astrophysics, and Direct Detection, JCAP 01 (2021) 039 [arXiv:2007.15563] [INSPIRE].

[4] I.M. Shoemaker and J. Wyenberg, Direct Detection Experiments at the Neutrino Dipole Portal Frontier, Phys. Rev. D 99 (2019) 075010 [arXiv:1811.12435] [INSPIRE].

[5] I.M. Shoemaker, Y.-D. Tsai and J. Wyenberg, An Active-to-Sterile Neutrino Transition Dipole Moment and the Xenon1T Excess, arXiv:2007.05513 [INSPIRE].

[6] R. Plestid, Luminous solar neutrinos I: Dipole portals, arXiv:2010.04193 [INSPIRE].

[7] P. Coloma, P.A.N. Machado, I. Martinez-Soler and I.M. Shoemaker, Double-Cascade Events from New Physics in IceCube, Phys. Rev. Lett. 119 (2017) 201804 [arXiv:1707.08573] [INSPIRE].

[8] S.N. Gninenko, The MiniBooNE anomaly and heavy neutrino decay, Phys. Rev. Lett. 103 (2009) 241802 [arXiv:0902.3802] [INSPIRE].

[9] S.N. Gninenko, A resolution of puzzles from the LSND, KARMEN, and MiniBooNE experiments, Phys. Rev. D 83 (2011) 015015 [arXiv: 1009.5536] [INSPIRE].

[10] M. Masip, P. Masjuan and D. Meloni, Heavy neutrino decays at MiniBooNE, JHEP 01 (2013) 106 [arXiv:1210.1519] [INSPIRE].

[11] P. Ballett, S. Pascoli and M. Ross-Lonergan, MeV-scale sterile neutrino decays at the Fermilab Short-Baseline Neutrino program, JHEP 04 (2017) 102 [arXiv:1610.08512] [INSPIRE].

[12] O. Fischer, A. Hernández-Cabezudo and T. Schwetz, Explaining the MiniBooNE excess by a decaying sterile neutrino with mass in the $250 \mathrm{MeV}$ range, Phys. Rev. D 101 (2020) 075045 [arXiv: 1909.09561] [INSPIRE].

[13] S. Vergani et al., Explaining the MiniBooNE Excess Through a Mixed Model of Oscillation and Decay, arXiv:2105.06470 [INSPIRE]. 
[14] SHIP collaboration, A facility to Search for Hidden Particles (SHiP) at the CERN SPS, arXiv: 1504.04956 [INSPIRE].

[15] J.L. Feng, I. Galon, F. Kling and S. Trojanowski, ForwArd Search ExpeRiment at the LHC, Phys. Rev. D 97 (2018) 035001 [arXiv:1708.09389] [INSPIRE].

[16] K. Jodłowski and S. Trojanowski, Neutrino beam-dump experiment with FASER at the LHC, JHEP 05 (2021) 191 [arXiv:2011.04751] [INSPIRE].

[17] D. Curtin et al., Long-Lived Particles at the Energy Frontier: The MATHUSLA Physics Case, Rept. Prog. Phys. 82 (2019) 116201 [arXiv:1806. 07396] [InSPIRE].

[18] MATHUSLA collaboration, An Update to the Letter of Intent for MATHUSLA: Search for Long-Lived Particles at the HL-LHC, arXiv:2009.01693 [INSPIRE].

[19] DUNE collaboration, Deep Underground Neutrino Experiment (DUNE), Far Detector Technical Design Report, Volume II: DUNE Physics, arXiv: 2002.03005 [INSPIRE].

[20] A.B. Balantekin, A. de Gouvêa and B. Kayser, Addressing the Majorana vs. Dirac Question with Neutrino Decays, Phys. Lett. B $\mathbf{7 8 9}$ (2019) 488 [arXiv:1808.10518] [INSPIRE].

[21] A. Baha Balantekin and B. Kayser, On the Properties of Neutrinos, Ann. Rev. Nucl. Part. Sci. 68 (2018) 313 [arXiv:1805.00922] [INSPIRE].

[22] J.M. Berryman, A. de Gouvêa, P.J. Fox, B.J. Kayser, K.J. Kelly and J.L. Raaf, Searches for Decays of New Particles in the DUNE Multi-Purpose Near Detector, JHEP 02 (2020) 174 [arXiv: 1912.07622] [INSPIRE].

[23] P. Coloma, E. Fernández-Martínez, M. González-López, J. Hernández-García and Z. Pavlovic, GeV-scale neutrinos: interactions with mesons and DUNE sensitivity, Eur. Phys. J. C 81 (2021) 78 [arXiv:2007.03701] [INSPIRE].

[24] M. Breitbach, L. Buonocore, C. Frugiuele, J. Kopp and L. Mittnacht, Searching for Physics Beyond the Standard Model in an Off-Axis DUNE Near Detector, arXiv:2102.03383 [INSPIRE].

[25] O. Tomalak and R.J. Hill, Theory of elastic neutrino-electron scattering, Phys. Rev. D 101 (2020) 033006 [arXiv:1907.03379] [INSPIRE].

[26] W.M. Haynes et al., eds., CRC Handbook of Chemistry and Physics, 97th edition, Taylor and Francis (2016).

[27] S. Sangiorgio et al., First demonstration of a sub-keV electron recoil energy threshold in a liquid argon ionization chamber, Nucl. Instrum. Meth. A 728 (2013) 69 [arXiv:1301.4290] [INSPIRE].

[28] P. Agnes et al., Measurement of the liquid argon energy response to nuclear and electronic recoils, Phys. Rev. D 97 (2018) 112005 [arXiv:1801.06653] [INSPIRE].

[29] C. Rubbia et al., Underground operation of the ICARUS T600 LAr-TPC: first results, 2011 JINST 6 P07011 [arXiv:1106.0975] [INSPIRE].

[30] ArgoNeuT collaboration, Measurement of $\nu_{\mu}$ and $\bar{\nu}_{\mu}$ neutral current $\pi^{0} \rightarrow \gamma \gamma$ production in the ArgoNeuT detector, Phys. Rev. D 96 (2017) 012006 [arXiv:1511.00941] [InSPIRE].

[31] MicroBooNE collaboration, First measurement of $\nu_{\mu}$ charged-current $\pi^{0}$ production on argon with the MicroBooNE detector, Phys. Rev. D 99 (2019) 091102 [arXiv:1811.02700] [INSPIRE]. 
[32] G.T. Garvey, D.A. Harris, H.A. Tanaka, R. Tayloe and G.P. Zeller, Recent Advances and Open Questions in Neutrino-induced Quasi-elastic Scattering and Single Photon Production, Phys. Rept. 580 (2015) 1 [arXiv:1412.4294] [INSPIRE].

[33] X. Zhang and B.D. Serot, Can neutrino-induced photon production explain the low energy excess in MiniBooNE?, Phys. Lett. B 719 (2013) 409 [arXiv:1210.3610] [InSPIRE].

[34] E. Wang, L. Álvarez-Ruso and J. Nieves, Photon emission in neutral current interactions at intermediate energies, Phys. Rev. C 89 (2014) 015503 [arXiv:1311.2151] [INSPIRE].

[35] T2K collaboration, Search for neutral-current induced single photon production at the ND280 near detector in T2K, J. Phys. G 46 (2019) 08LT01 [arXiv:1902.03848] [INSPIRE].

[36] DUNE collaboration, Deep Underground Neutrino Experiment (DUNE), Far Detector Technical Design Report, Volume I Introduction to DUNE, 2020 JINST 15 T08008 [arXiv: 2002.02967] [INSPIRE].

[37] L. Fields, CP Optimized Beam, Neutrino Mode, June 2020, https://home.fnal.gov/ ljf26/DUNEFluxes/OptimizedEngineeredNov2017_offaxis/.

[38] A.M. Dziewonski and D.L. Anderson, Preliminary reference earth model, Phys. Earth Planet. Interiors 25 (1981) 297.

[39] DUNE collaboration, Deep Underground Neutrino Experiment (DUNE), Far Detector Technical Design Report, Volume IV: Far Detector Single-phase Technology, 2020 JINST 15 T08010 [arXiv: 2002.03010] [INSPIRE].

[40] SHIP collaboration, Measurement of the muon flux for the SHiP experiment, arXiv:2001.04784 [INSPIRE].

[41] DUNE collaboration, Deep Underground Neutrino Experiment (DUNE) Near Detector Conceptual Design Report, arXiv:2103.13910 [INSPIRE].

[42] R.L. Donahue, R.W. Miller and J.C. Shickluna, Soils: an introduction to soils and plant growth, 4th ed., Englewood, Cliffs, N.J. eds., Prentice-Hall (1977) https://archive.org/details/soilsintroductio00dona.

[43] SHIP collaboration, Sensitivity of the SHiP experiment to Heavy Neutral Leptons, JHEP 04 (2019) 077 [arXiv : 1811.00930] [INSPIRE].

[44] C. Giunti and A. Studenikin, Neutrino electromagnetic interactions: a window to new physics, Rev. Mod. Phys. 87 (2015) 531 [arXiv:1403.6344] [InSPIRE].

[45] C. Giunti, K.A. Kouzakov, Y.-F. Li, A.V. Lokhov, A.I. Studenikin and S. Zhou, Electromagnetic neutrinos in laboratory experiments and astrophysics, Annalen Phys. 528 (2016) 198 [arXiv : 1506.05387] [INSPIRE].

[46] CHARM-II collaboration, A New Determination of the Electroweak Mixing Angle From $\nu_{\mu}$ Electron Scattering, Phys. Lett. B 232 (1989) 539 [INSPIRE].

[47] S.N. Gninenko and N.V. Krasnikov, Limits on the magnetic moment of sterile neutrino and two photon neutrino decay, Phys. Lett. B 450 (1999) 165 [hep-ph/9808370] [INSPIRE].

[48] DONUT collaboration, A New upper limit for the tau-neutrino magnetic moment, Phys. Lett. B 513 (2001) 23 [hep-ex/0102026] [INSPIRE].

[49] S. Alekhin et al., A facility to Search for Hidden Particles at the CERN SPS: the SHiP physics case, Rept. Prog. Phys. 79 (2016) 124201 [arXiv: 1504.04855] [InSPIRE]. 
[50] W. Grimus, M. Maltoni, T. Schwetz, M.A. Tortola and J.W.F. Valle, Constraining Majorana neutrino electromagnetic properties from the LMA-MSW solution of the solar neutrino problem, Nucl. Phys. B 648 (2003) 376 [hep-ph/0208132] [InSPIRE].

[51] BoreXINo collaboration, Limiting neutrino magnetic moments with Borexino Phase-II solar neutrino data, Phys. Rev. D 96 (2017) 091103 [arXiv:1707.09355] [INSPIRE].

[52] Borexino collaboration, Comprehensive measurement of pp-chain solar neutrinos, Nature 562 (2018) 505 [INSPIRE].

[53] SuPer-Kamiokande collaboration, Solar Neutrino Measurements in Super-Kamiokande-IV, Phys. Rev. D 94 (2016) 052010 [arXiv: 1606. 07538] [inSPIRE].

[54] A. Atre, T. Han, S. Pascoli and B. Zhang, The Search for Heavy Majorana Neutrinos, JHEP 05 (2009) 030 [arXiv:0901.3589] [INSPIRE].

[55] K. Bondarenko, A. Boyarsky, D. Gorbunov and O. Ruchayskiy, Phenomenology of GeV-scale Heavy Neutral Leptons, JHEP 11 (2018) 032 [arXiv:1805.08567] [INSPIRE].

[56] R. Plestid, Luminous solar neutrinos II: Mass-mixing portals, arXiv:2010.09523 [INSPIRE].

[57] P. Coloma, P. Hernández, V. Muñoz and I.M. Shoemaker, New constraints on Heavy Neutral Leptons from Super-Kamiokande data, Eur. Phys. J. C 80 (2020) 235 [arXiv:1911.09129] [INSPIRE].

[58] J. Orloff, A.N. Rozanov and C. Santoni, Limits on the mixing of tau neutrino to heavy neutrinos, Phys. Lett. B 5550 (2002) 8 [hep-ph/0208075] [INSPIRE].

[59] DELPHI collaboration, Search for neutral heavy leptons produced in $Z$ decays, Z. Phys. C 74 (1997) 57 [Erratum ibid. 75 (1997) 580] [INSPIRE].

[60] A.D. Dolgov, S.H. Hansen, G. Raffelt and D.V. Semikoz, Heavy sterile neutrinos: Bounds from big bang nucleosynthesis and SN1987A, Nucl. Phys. B $\mathbf{5 9 0}$ (2000) 562 [hep-ph/0008138] [INSPIRE].

[61] G.G. Raffelt, Particle physics from stars, Ann. Rev. Nucl. Part. Sci. 49 (1999) 163 [hep-ph/9903472] [INSPIRE].

[62] A.D. Dolgov, S.H. Hansen, G. Raffelt and D.V. Semikoz, Cosmological and astrophysical bounds on a heavy sterile neutrino and the KARMEN anomaly, Nucl. Phys. B 580 (2000) 331 [hep-ph/0002223] [INSPIRE].

[63] M. Atkinson, P. Coloma, I. Martinez-Soler, N. Rocco and I.M. Shoemaker, Heavy Neutrino searches through Double-Bang Events at Super-Kamiokande, DUNE, and Hyper-Kamiokande, arXiv:2105.09357 [INSPIRE].

[64] J.D. Lewin and P.F. Smith, Review of mathematics, numerical factors, and corrections for dark matter experiments based on elastic nuclear recoil, Astropart. Phys. 6 (1996) 87 [INSPIRE].

[65] P. Huber, J. Kopp, M. Lindner, M. Rolinec and W. Winter, New features in the simulation of neutrino oscillation experiments with GLoBES 3.0: General Long Baseline Experiment Simulator, Comput. Phys. Commun. 177 (2007) 432 [hep-ph/0701187] [InSPIRE].

[66] M. Galassi et al., GNU Scientific Library Reference Manual, 3rd edition, GNU http://www.gnu.org/software/gsl/. 